OPEN ACCESS

Edited by: Michele Papa,

University of Campania Luigi

Vanvitelli, Italy

Reviewed by:

Ying Zhang,

Dalhousie University, Canada Marina Boido,

University of Turin, Italy

*Correspondence: Frédéric Clotman frederic.clotman@uclouvain.be

Received: 06 June 2019 Accepted: 16 October 2019 Published: 07 November 2019

Citation:

Masgutova G, Harris A, Jacob B, Corcoran LM and Clotman F (2019) Pourf2 Regulates the Distribution of Dorsal Interneurons in the Mouse Developing Spinal Cord.

Front. Mol. Neurosci. 12:263. doi: 10.3389/fnmol.2019.00263

\section{Pou2f2 Regulates the Distribution of Dorsal Interneurons in the Mouse Developing Spinal Cord}

\author{
Gauhar Masgutova ${ }^{1}$, Audrey Harris ${ }^{1}$, Benvenuto Jacob ${ }^{2}$, Lynn M. Corcoran ${ }^{3}$ \\ and Frédéric Clotman ${ }^{1 *}$ \\ ${ }^{1}$ Université catholique de Louvain, Institute of Neuroscience, Laboratory of Neural Differentiation, Brussels, Belgium, \\ ${ }^{2}$ Université catholique de Louvain, Institute of Neuroscience, System and Cognition Division, Brussels, Belgium, ${ }^{3}$ Molecular \\ Immunology Division and Immunology Division, The Walter and Eliza Hall Institute, Parkville, VIC, Australia
}

Spinal dorsal interneurons, which are generated during embryonic development, relay and process sensory inputs from the periphery to the central nervous system. Proper integration of these cells into neuronal circuitry depends on their correct positioning within the spinal parenchyma. Molecular cues that control neuronal migration have been extensively characterized but the genetic programs that regulate their production remain poorly investigated. Onecut (OC) transcription factors have been shown to control the migration of the dorsal interneurons (dINs) during spinal cord development. Here, we report that the $\mathrm{OC}$ factors moderate the expression of Pou2f2, a transcription factor essential for B-cell differentiation, in spinal dINs. Overexpression or inactivation of Pou2f2 leads to alterations in the differentiation of $\mathrm{dl} 2$, dl3 and Phox2a-positive dl5 populations and to defects in the distribution of dl2-dl6 interneurons. Thus, an OC-Pou2f2 genetic cascade regulates adequate diversification and distribution of dINs during embryonic development.

Keywords: embryonic spinal cord, dorsal interneurons, Pou2f2, onecut, neuronal migration

\section{INTRODUCTION}

The dorsal spinal cord relays and processes somatosensory inputs, nociception, thermosensation, pruriception, mechanosensation and proprioception, from peripheral sensory neurons to central targets. Furthermore, somatosensory perception is crucial for fine regulation and proper execution of motor activities. The complex organization of these neural circuits requires precise spatial position of neuronal cell bodies and integration into proper connectivity routes (Lu et al., 2015; Lai et al., 2016). Neuronal positioning and the cellular and molecular mechanisms involved in this process have been extensively studied in the developing brain (Marín et al., 2010). Although the molecules directing neuronal migration in the developing spinal cord start to be identified (Andrews et al., 2006; Kim et al., 2015; Junge et al., 2016; Leggere et al., 2016), much less is known about the genetic programs that control this essential process.

During embryonic development, eight populations of dorsal interneurons (dINs) are produced from progenitor domains orderly distributed along the dorso-ventral (DV) 
axis of the ventricular zone in the dorsal spinal cord. A unique combinatorial code of basic helix-loop-helix (bHLH) or homeodomain transcription factors defines, in two neurogenic waves, six early dIN populations (dI1-dI6) generated between embryonic day (e) 10.5 and e12.5, and two late-born $\mathrm{dIL}^{\mathrm{A}}$ and $\mathrm{dIL}^{\mathrm{B}}$ INs produced between e11 and e13 (Caspary and Anderson, 2003; Helms and Johnson, 2003). When specified, dINs migrate long distance along the DV axis to their distinctive location and integrate into specific circuits (Hernandez-Miranda et al., 2017). Among the early-born dINs, the dI1 excitatory INs localize in the intermediate spinal cord (Bermingham et al., 2001) while some dI2 excitatory INs migrate towards the intermediate layers of the spinal cord and others invade the ventral horn (Gross et al., 2002). The intermediate and deep dorsal horn contains glutamatergic dI3 (Bui et al., 2013). The inhibitory dI4 INs settle laterally in the deep dorsal horn (Gross et al., 2002; Müller et al., 2002; Pillai et al., 2007) and excitatory dI5 INs invade the deep dorsal and the ventral horns (Ding et al., 2004). Located in the ventromedial region of the spinal cord, dI6 INs give rise to Dmrt3- or WT1-containing subsets (Lanuza et al., 2004; Andersson et al., 2012; Schnerwitzki et al., 2018). The two late-born $\mathrm{dIL}^{\mathrm{A}}$ and $\mathrm{dIL}^{\mathrm{B}}$ populations occupy the superficial laminae of the dorsal horn (Gross et al., 2000; Müller et al., 2002). During spinal cord development, these heterogeneous dorsal populations continue to diversify into small and discrete subsets (Ding et al., 2004; Del Barrio et al., 2013; Rosenberg et al., 2018) that each follows a stereotyped pattern of migration to reach their final location within the spinal parenchyma.

Spinal neuron distribution along the DV and mediolateral (ML) axes constitute a critical feature of microcircuit organization and functionality (Ladle et al., 2007; Tripodi et al., 2011). Indeed, proper cell body position of dorsal inhibitory INs along the ML axis is crucial for the establishment of their sensorimotor connectivity (Hilde et al., 2016) while the distribution of distinct Lbx1-positive premotor (Goetz et al., 2015) or V1 IN subsets (Bikoff et al., 2016) constrains patterns of input from sensory and motor neurons. In addition, V3 INs segregate into dorsal or ventral sub-populations that differ in their connectivity patterns and are active during distinct motor activities (Borowska et al., 2013, 2015; Chopek et al., 2018). Furthermore, segmental distinctions exist along the rostro-caudal axis of the spinal cord. Columnar organization of motor neurons varies between brachial or lumbar and thoracic regions in register with the part of the body these cells target (Francius and Clotman, 2014; Catela et al., 2015). In parallel, as several INs populations are associated with differential motor output, IN integration into local microcircuits is also influenced by their distribution along the antero-posterior axis (Bikoff et al., 2016; Hayashi et al., 2018; Sweeney et al., 2018). Even if the genetic networks orchestrating the production and the differentiation of spinal INs have been extensively deciphered (Lu et al., 2015), less is known about the transcription factors that dictate their distribution in the developing spinal cord and might consequently influence their connectivity profiles and spinal circuit formation. The Onecut (OC) transcription factors namely Hepatocyte Nuclear Factor-6 (HNF-6, or OC-1), OC- 2 and OC-3, are detected in the digestive tract and in the
CNS during embryonic development (Lemaigre et al., 1996; Landry et al., 1997; Jacquemin et al., 1999, 2003; Vanhorenbeeck et al., 2002). Besides their roles in the production (Espana and Clotman, 2012b), diversification (Roy et al., 2012; Francius and Clotman, 2014; Kabayiza et al., 2017) or maintenance (Espana and Clotman, 2012a,b; Stam et al., 2012) of specific neuronal populations, they also regulate neuronal distribution of various neuron types. OC regulate proper organization and migration of dopaminergic neurons of the A13 nucleus during their second phase of development (Espana and Clotman, 2012b). Similarly, they contribute to the reorganization of the Purkinje cells during a late phase of cerebellar development (Audouard et al., 2013). More recently, their contribution in the regulation of neuronal distribution was also uncovered in dorsal and in ventral spinal INs (Kabayiza et al., 2017; Harris et al., 2019). However, little is known about the downstream molecular effectors of the OC factors in dIN development, particularly regarding their migration along the DV and the ML axes of the spinal cord. In the present study, we uncovered that the expression of Pou2f2, a POU family transcription factor (Clerc et al., 1988; Corcoran et al., 1993), is controlled by OC factors in the spinal dINs. We show that Pou2f2 is present in dI2-dI6 populations during the early stages of development. Analysis of OC mutant embryos demonstrated that OC proteins moderate the expression of Pou $2 f 2$ in the dorsal spinal cord. Using gain-of-function experiments, we observed that increased Pou2f2 modulates the distribution of the dI2-dI6 INs. Lossof-function experiments confirmed that Pou $2 \mathrm{f} 2$ regulates the localization of the dI2-dI6 and the number of cells in some dIN populations. Thus, Pou2f 2 controls the differentiation and the distribution of dINs in the developing spinal cord.

\section{MATERIALS AND METHODS}

\section{Ethic Statement and Mouse Lines}

All experiments were strictly performed in accordance with the European Community Council directive of 24 November 1986 (86-609/ECC) and the decree of 20 October 1987 (87-848/EEC). Mice were raised in our animal facilities and treated according to the principles of laboratory animal care. Experimental procedures and mouse housing were both approved by the Animal Welfare Committee of Université Catholique de Louvain (Permit Numbers: 2013/UCL/MD/11 and 2017/UCL/MD/008). Mutant strain mice were crossed and the day of vaginal plug was considered to be embryonic day (e) 0.5 . The embryos were collected at e10.5, e11.5, e12.5 and e14.5. A minimum of three embryos of the same genotype was analyzed in each experiment. The Hnf6;Oc2 and the Pou $2 f 2$ mutant mice have been described previously (Corcoran et al., 1993; Jacquemin et al., 2000; Clotman et al., 2005). The Hnf6;Oc2 mutant embryos additionally lack OC-3 in the developing spinal cord (Roy et al., 2012; Kabayiza et al., 2017).

\section{In situ Hybridization and Immunofluorescence Labelings}

For in situ hybridization, embryos were fixed in ice-cold $4 \%$ paraformaldehyde (PFA) in phosphate buffered-saline (PBS) 
overnight at $4^{\circ} \mathrm{C}$, washed thrice in PBS for $10 \mathrm{~min}$, incubated in $\mathrm{PBS} / 30 \%$ sucrose solution overnight at $4^{\circ} \mathrm{C}$, embedded and frozen in $\mathrm{PBS} / 15 \%$ sucrose $/ 7.5 \%$ gelatin. Fourteen micrometer section were prepared and in situ hybridization was performed as previously described (Beguin et al., 2013; Pelosi et al., 2014; Francius et al., 2016) with DIG-conjugated Pou2f2 (NM_011138.1, nucleotides 604-1,187) antisense RNA probes. Control or $\mathrm{Hnf6/Oc2} 2^{-/-}$mutant sections were placed adjacent on the same histology slides to minimize inter-slide variations of in situ hybridization signals. For immunofluorescence labelings, embryos were fixed in ice-cold 4\% PFA in PBS for 10-35 min according to their embryonic stage, incubated in $\mathrm{PBS} / 30 \%$ sucrose solution overnight at $4^{\circ} \mathrm{C}$, embedded and frozen in $\mathrm{PBS} / 15 \%$ sucrose $/ 7.5 \%$ gelatin. Immunostaining was performed on 12 or $14 \mu \mathrm{m}$ serial cryosections as previously described (Francius and Clotman, 2010). Primary antibodies against the following proteins were used: Brn3a (mouse 1:1,000; Santa Cruz \#sc-8429), Dmrt3 (guinea pig; 1:1,000; kindly provided by K. Kullander \#170), Foxd3 (guinea pig; 1:1,000; or rabbit; 1:1,000; kindly provided by T. Müller), Foxp1 (goat; 1:1,000; R\&D Systems \#AF4534), HNF6 [guinea pig; 1:2,000; (Espana and Clotman, 2012a) or rabbit; 1:100; Santa Cruz \#sc-13050 or sheep; 1:1,000 R\&D Systems \#AF6277], Isl1/2 (goat; 1:3,000; Neuromics \#GT15051 or mouse; 1:6,000; DSHB \#39.4D5), Lbx1 (guinea pig; 1:10,000 or rabbit; 1:5,000; kindly provided by T. Müller), Lhx1/5 (mouse; 1:1,000; DSHB \#4F2), Lmx1b (guinea pig; 1:10,000 or rabbit; 1:2,000; kindly provided by T. Müller), OC2 [rat; 1:400; (Clotman et al., 2005) or sheep; 1:500; R\&D Systems \#AF6294], OC3 [guinea pig; 1:6,000; (Pierreux et al., 2004), Phox2a (rabbit; 1:500; kindly provided by J.-F. Brunet), Pou2f2 (rabbit; 1:2,000; Abcam \#ab178679), Wt1 (rabbit; 1:2,000; Santa Cruz \#sc-192)]. Secondary antibodies were the donkey anti-guinea pig/AlexaFluor 488, 594 or 647, anti-mouse/AlexaFluor 488, 594 or 647, antirabbit/AlexaFluor 594 or 647, anti-goat/AlexaFluor 488, anti-rat/AlexaFluor 488 or 647, anti-sheep/AlexaFluor 594, and goat anti-mouse IgG1 specific/AlexaFluor 488 or 594, anti-mouse IgG2A specific/AlexiaFluor 488, anti-mouse IgG2B specific/AlexaFluor 647, purchased from Thermo Fisher Scientific or Jackson Laboratories, and were used at dilution 1:1,000.

\section{In ovo Electroporation}

In ovo electroporations were performed at stage HH14-16, as previously described (Roy et al., 2012). The coding sequence of Pou $2 f 2$ was amplified by overlapping-PCR, as previously described Harris using: forward $5^{\prime}$ GCTCTGTCTGCC CAAGAGAAA $3^{\prime}$ and reverse $5^{\prime}$ GTTGGGACAAGGTGA GCTGT $3^{\prime}$ primers for the $5^{\prime}$ region, forward $5^{\prime}$ CCACC ATCACAGCCTACCAG $3^{\prime}$ and reverse $5^{\prime}$ ATTATCTCG AGCCAGCCTCCTTACCCTCTCT $3^{\prime}$ (designed to enable integration at the XhoI restriction site of the pCMV-MCS vector) primers for the $3^{\prime}$ region. This sequence was first subcloned in a pCR ${ }^{\circledR} \mathrm{II}-$ Topo ${ }^{\circledR}$ vector (Life Technologies, 45-0640) for sequencing then subcloned at the EcoRI (from the pCR ${ }^{\circledR}$ II-Topo ${ }^{\circledR}$ vector) and XhoI restriction sites of a pCMV-MCS vector for the in ovo electroporation.
The pCMV-Pou2f2 $(0.5 \mu \mathrm{g} / \mu \mathrm{l})$ vector was co-electroporated with a pCMV-eGFP plasmid $(0.25 \mu \mathrm{g} / \mu \mathrm{l})$ to visualize electroporated cells. The embryos were collected $72 \mathrm{~h}$ (HH27-28) after electroporation, fixed in $\mathrm{PBS} / 4 \% \mathrm{PFA}$ for $45 \mathrm{~min}$ and processed for immunofluorescence labelings as previously described (Francius and Clotman, 2010).

\section{In situ Hybridization Signal Intensity Measurements}

In situ hybridization images of cryosections were acquired on an EVOS FL Auto Cell Imaging System (Thermo Fisher Scientific, Waltham, MA, USA). For each embryo $(n=3)$, one side of five sections at lumbar level was quantified using ImageJ by delineating an area of $13,500 \mathrm{px}^{2}$ using the "rectangular" selection tool and evaluating signal intensity using Measure under Analyze. Intensity of the background signal from an adjacent area devoid of labeling was subtracted to normalize for background variations. All numbers are arbitrary units. Raw data were exported from ImageJ software to Sigma Plot v12.3 software to perform statistical analysis. The histograms were drawn with Microsoft Excel. Adequate statistical test was applied based on the number of comparisons and on the variance in each group. For analysis of signal intensity based on comparison of two groups (control or $\mathrm{Hnf} 6 / \mathrm{Oc} 2^{-/-}$), a standard Student's $t$-test was performed. Difference was considered as significant at $p \leq 0.05$.

\section{Cell Quantification}

Immnofluorescence images of cryosections were acquired on a Confocal Laser Scanning microscope FV1000 Fluoview with the FV10-ASW 01.02 software (Olympus) or an EVOS FL Auto Cell Imaging System (Thermo Fisher Scientific, Waltham, MA, USA). For each labeling, acquisition parameters were identical for control or mutant sections. Brightness and contrast were adjusted uniformly in all replicate panels within an experiment with Adobe Photoshop CS6 software to match with the observation. Quantifications were performed on red or green or blue layer of acquired confocal images and double or triple labeled cells were processed by subtractive method (Francius and Clotman, 2010). For each embryo $(n=3)$, one side of five sections at brachial, at thoracic and at lumbar levels were quantified using the count analysis tool of Adobe Photoshop CS6 software. Raw data were exported from Adobe Photoshop CS6 software to Sigma Plot v12.3 software to perform statistical analyses, and the histograms were drawn with Microsoft Excel. Adequate statistical tests were applied based on the number of comparisons and on the variance in each group. For analysis of cell quantifications based on comparison of two groups (control or mutant; control or electroporated side of the spinal cord), standard Student's $t$-tests or Mann-Whitney $U$ tests were performed. Differences were considered as significant at $p \leq 0.05$.

\section{Spatial Distribution}

Pictures for quantitative analyses of dorsal interneuron distribution were acquired on a Confocal Laser Scanning microscope FV1000 Fluoview with the FV10-ASW 
01.02 software (Olympus). Spinal cord height $(\mathrm{H})$ was defined as the distance from the ventral-most to the dorsal-most point of spinal cord, and width (W) as the distance from central canal to the most lateral edge (adapted from Palmesino et al., 2010). For each dIN, distance $\left(D_{I N}\right)$ and angle $\left(\alpha_{I N}\right)$ were measured from the ventral-most point of the spinal cord to the interneuron soma using the ruler analysis tool in Adobe Photoshop CS6 software. DV and ML position of dINs were expressed as percentage of spinal cord height and hemicord width, respectively: DV position and ML position were defined as $\left(D_{I N} * \sin \alpha_{I N}\right) / H$ and $\left(D_{I N} * \cos \alpha_{I N}\right) / \mathrm{W}$, respectively (Palmesino et al., 2010). ML vs. DV values were plotted using Matlab software R2013a (Mathworks, Canada). Statistical analyses of dIN distribution were performed using a two-sample Hotelling's T2, which is a two-dimensional generalization of the Student's $t$-test, as described for similar data sets (Palmesino et al., 2010; Kabayiza et al., 2017; Harris et al., 2019). The analysis was implemented using the NCSS software package.

\section{RESULTS}

\section{OC Factors Moderate Pou2f2 Expression in dIN Populations}

The OC transcription factors control the distribution of dIN populations (Kabayiza et al., 2017). In an effort to identify genes downstream of OC involved in this process, we analyzed the results of a transcriptomic comparison between embryonic day (e)11.5 wildtype or OC-deficient spinal cords (GEO repository accession number: GSE117871; Harris et al., 2019). Surprisingly, we could not identify significant changes in expression of genes coding for demonstrated or potential migration cues, except for Draxin. However, expression pattern of Draxin or distribution of the corresponding protein was not changed in $O C$ mutant spinal cords (data not shown). In contrast, we confirmed that the expression level of Pou $2 f 2$, a transcription factor containing a POU-specific domain and a POU-type homeodomain, was significantly increased in the spinal cord in the absence of OC proteins. Pou $2 f 2$ is expressed in B-lymphocytes, in neuronal cell lines and in neural tissues (Hatzopoulos et al., 1990; Lillycrop and Latchman, 1992; Camós et al., 2014). It is required for differentiation of $\mathrm{B}$ lymphocytes and is able to modulate neuronal differentiation of ES cells (Corcoran et al., 1993, 2004; König et al., 1995; Theodorou et al., 2009; Hodson et al., 2016). The expression level of Pou2f2 was 2.6-fold upregulated in $\mathrm{Hnf6/Oc2^{-/ }}$ spinal cords (Harris et al., 2019). However, a potential contribution of Pou2f2 to dIN development was unknown.

Using in situ hybridization, we first confirmed that Pou $2 f 2$ expression was increased in the dorsal part of the spinal cord in $\mathrm{Hnf6/Oc2^{-/- }}$ embryos. Consistent with quantifications on the whole spinal cord (Harris et al., 2019), we measured a $\sim 3$-fold increase in signal intensity in the absence of OC proteins (Figure 1). To assess the distribution of Pou $2 \mathrm{f} 2$ in the dIN populations and to determine if the increase in Pou 2 f2 expression was due to an expansion of Pou2f2 distribution or to an upregulation in its endogenous expression domain, we quantified the number of Pou2f2-containing dINs between e10.5 and e12.5 in wildtype and in OC-deficient embryos. As previously demonstrated, the $\mathrm{OC}$ factors are neither present in dI1 nor in dIL ${ }^{A}$ and dIL $^{B}$ INs (Kabayiza et al., 2017). Moreover, due to the lack of antibodies for dI4 and early dI6 specific markers compatible with the Pou2f2 antibody species (see "Materials and Methods" section), we were unable to study the presence of Pou2f 2 in those populations.

Immunofluorescence analyses demonstrated that Pou2f 2 is produced in post-mitotic dI 2 INs, defined by the presence of
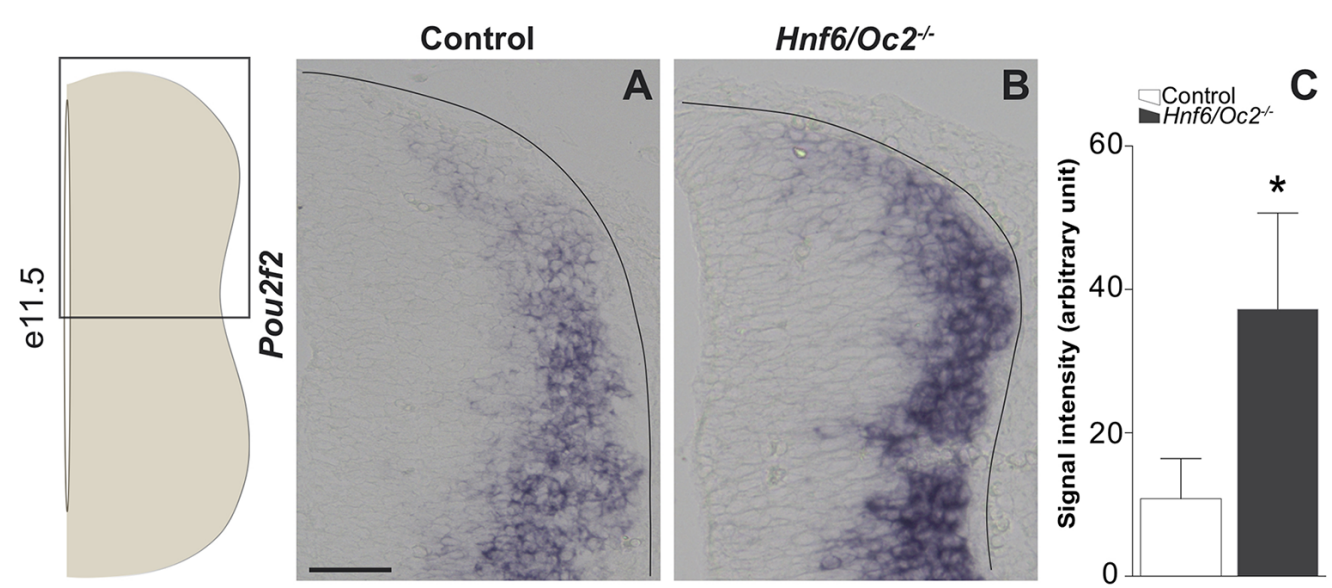

FIGURE 1 | Onecut (OC) factors moderate expression of Pou2f2 in the dorsal spinal cord. (A,B) In situ hybridization for Pou2f2 on transverse sections (lumbar level) of control or Hnf6/Oc2 ${ }^{-1-}$ spinal cords at e11.5. (A) In control embryos, Pou2f2 is expressed at low levels in the dorsal part of the spinal cord and at higher levels in the intermediate zone. (B) In Hnf6/Oc2 ${ }^{-/-}$mutant embryos, Pou2f2 expression is upregulated and cells displaying high Pou2f2 levels are observed more dorsally than in control littermates. The pictures show part of right hemisections as indicated on the scheme to the left. (C) Measurement of Pou2f2 in situ hybridization signal intensity in control or Hnf6/Oc2 $2^{-1-}$ at e11.5. Pou2f2 expression is upregulated in the absence of the OC factors in the dorsal spinal cord $(p \leq 0.05)$. Mean values $\pm \operatorname{SEM}, n=3 .{ }^{*}=p \leq 0.05$. Solid lines delineate the spinal cord. Scale bar $=100 \mu \mathrm{m}$ 
Foxd 3 at e10.5 and e11.5 and of Foxd3 and Brn3a at e12.5. In control embryos, Pou2f2 was detected in a significant fraction of a ventral dI2 cell contingent at e10.5 (Figures 2A,B) but, as observed for OC factors (Kabayiza et al., 2017), the proportion of dI2 Pou2f2-positive INs decreased at e11.5 (Figures 2C,D) and Pou2f2 was almost completely absent from dI 2 cells at e12.5 (Figures 2E,F). In mutant embryos, the number of Pou2f2positive dI2 trended to increase as compared to control embryos, but this change was not statistically significant. Labeling intensity was stronger in dI2 of OC mutant embryos (Figures 2A-F), consistent with the increased Pou2f2 expression in the dorsal regions of the spinal cord.

In dI3 INs, characterized by the presence of Isl1, Pou2f2 was detected in the ventral part of the population from the early stages of their development (Figures 3A,B). As observed for $\mathrm{dI} 2 \mathrm{INs}$, the proportion of Pou2f2-positive $\mathrm{dI} 3$ cells progressively decreased as development proceeds (Figures 3C-I). Pou2f2 was detected in a similar proportion of $\mathrm{dI} 3$ cells in control and in mutant embryos between e10.5 and e12.5 (Figures 3G-I).
Nonetheless, the signal intensity was stronger in $O C$ mutant dI3 cells (Figures 3A-F).

Lmx1b early-born dI5 INs were analyzed at e10.5 and e11.5 to distinguish them from late-born $\mathrm{dIL}^{\mathrm{B}}$ also identified by Lmx $1 \mathrm{~b}$ at later stages. In control embryos, as for the other dIN populations, Pou2f2-positive cells represented a higher proportion of dI5 INs at e10.5, with most of the dI5 containing Pou2f2 (Figure 4A), while this proportion decreased at e11.5 (Figure $4 \mathrm{C}$ ). In $\mathrm{Hnf6} / \mathrm{Oc} 2^{-/-}$mutant embryos, the proportion of Pou2f2positive dI5 cells trended to decrease at e10.5 (Figures 4A-C) and to increase at e11.5 (Figures 4D-F). However, these changes were not statistically significant (Figures 4C,F). Again, stronger intensity of the labeling at e11.5 confirmed increased Pou2f 2 production in dI5 INs.

The lack of a unique specific marker for dI6 INs complicates the analysis of Pou2f2 in this population at early stages. Nevertheless, we were able to characterize the presence of Pou2f2 in the dI6 Dmrt3 ${ }^{+}$subset at e12.5 (Figures 4G-I). In control embryos, between $20 \%$ and $40 \%$ of dI6 cells
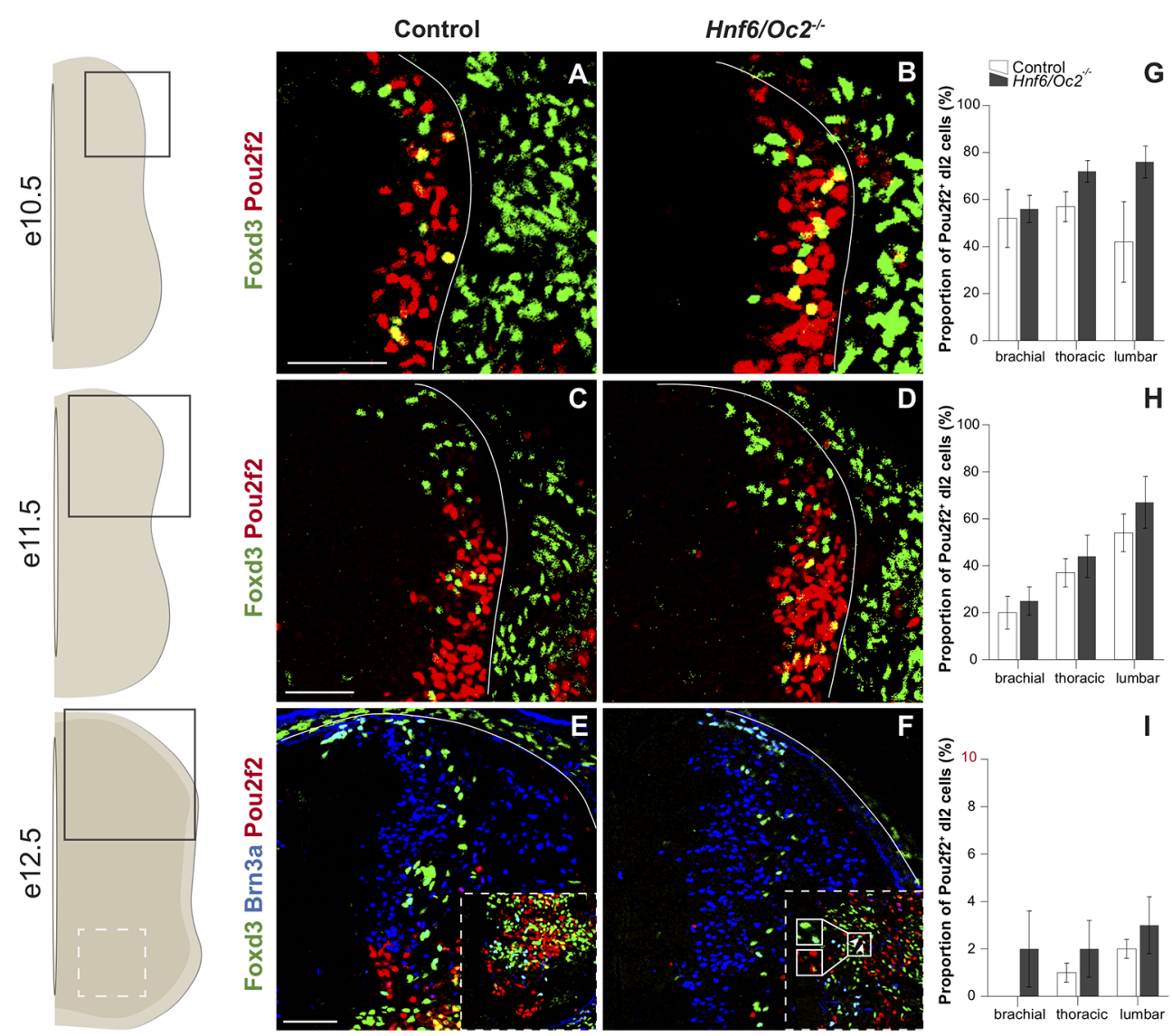

FIGURE 2 | The OC factors moderate Pou2f2 expression in dl2 interneurons. (A-F) Immunodetection of Pou2f2 (red) in Foxd3 ${ }^{+}$(green) or Foxd3 ${ }^{+}$(green) Brn3a ${ }^{+}$ (blue) dl2 interneurons on transverse sections of thoracic spinal cord at e10.5 (A,B), e11.5 (C,D) and e12.5 (E,F). Pou2f2 is detected in post-mitotic Foxd3 ${ }^{+}$ $\mathrm{dl} 2$ interneurons, particularly in the ventral part of the population, at e10.5 and e11.5 but is almost absent at e12.5. Pou2f2 signal is stronger in Foxd $3^{+}$ dl2 interneurons in $\mathrm{Hnf6/Oc2}{ }^{-/-}$spinal cord at all studied developmental stages. (G-I) Relative quantification of Pou2f2 positive dl2 neurons at e10.5 (G), e11.5

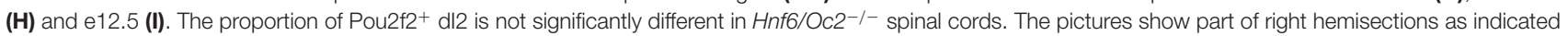
on the schemes to the left. Solid lines delineate the spinal cord. Insets in (E,F) are magnified views of boxed ventral regions. Arrowheads in (F) point to triple-labeled cells. Mean values \pm SEM, $n=3$. Scale bars $=100 \mu \mathrm{m}$. 

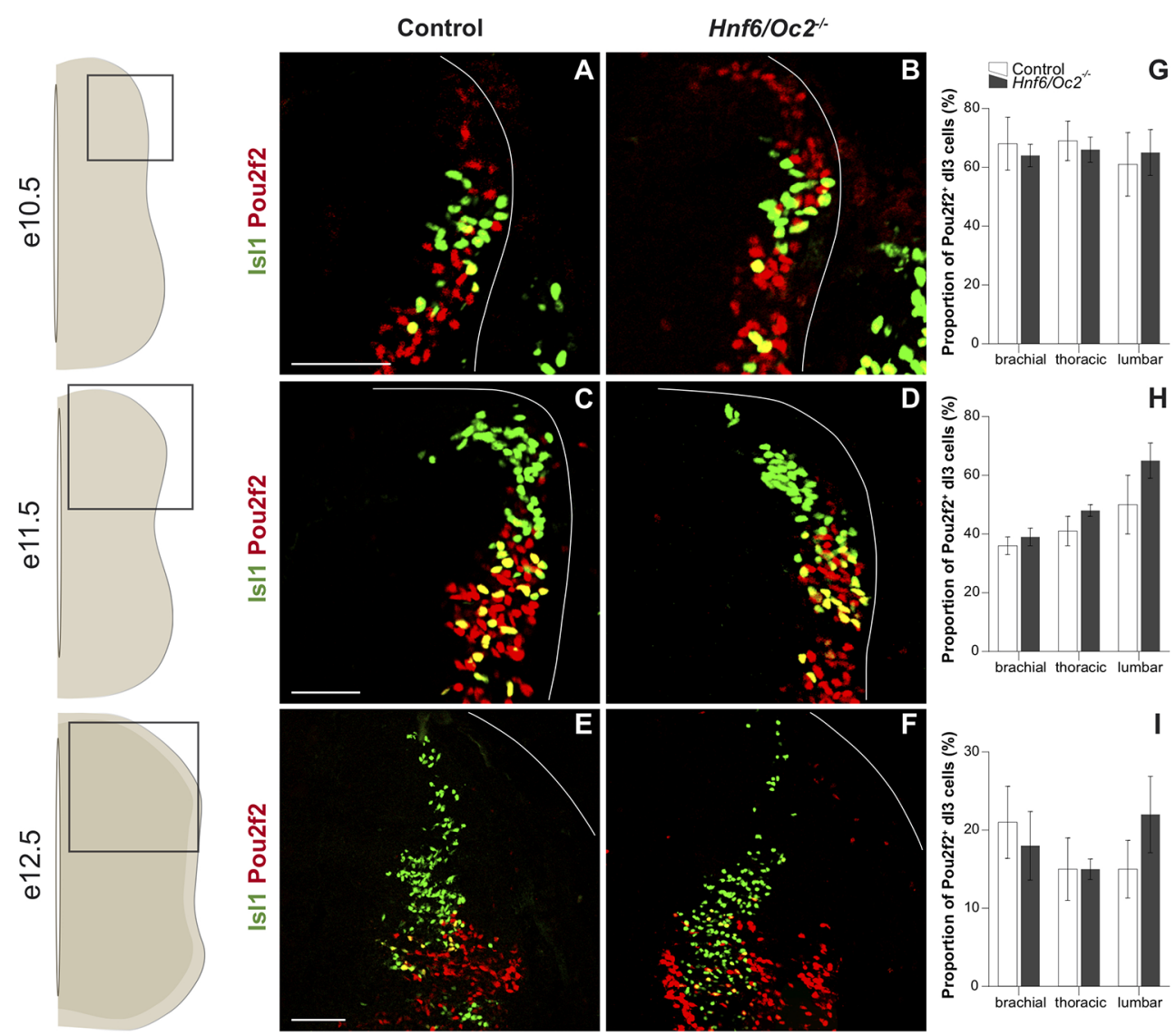

FIGURE 3 | The OC factors moderate Pou2f2 expression in dl3 interneurons. (A-F) Immunodetection of Pou2f2 (red) in Isl1+ (green) dl3 interneurons on transverse sections of thoracic spinal cord at e10.5 (A,B), at e11.5 (C,D) and e12.5 (E,F). Pou2f2 is detected in the ventral part of the post-mitotic Isl1+ dl3 interneuron population from e10.5. Pou2f2 signal is stronger in Isl1+ $\mathrm{dl3}$ interneurons in Hnf6/Oc2 ${ }^{-/-}$spinal cord at all studied developmental stages. (G-I) Relative quantification of Pou2f2 positive dl3 neurons at e10.5 (G), e11.5 (H) and e12.5 (I). The proportion of Pou2f2 ${ }^{+}$dl3 is not significantly different in Hnf6/Oc2 ${ }^{-/-}$spinal cords. The pictures show part of right hemisections as indicated on the schemes to the left. Solid lines delineate the spinal cord. Mean values \pm SEM, $n=3$. Scale bars $=100 \mu \mathrm{m}$

contained Pou2f2. The proportion of dI6 Dmrt3 ${ }^{+}$producing Pou2f2 trended to increase in mutant compared to control embryos, although the change was statistically significant only at thoracic level (Figure 4I). Moreover, as for the other dorsal populations, Pou2f2 signal was stronger in $\mathrm{Hnf6/Oc2} 2^{-/-}$mutant embryos. Taken together, these observations suggest that $\mathrm{OC}$ factors temper Pou2f2 expression in dINs and restrain its expression to a subset of dI6 INs.

\section{Pou2f2 Overexpression Alters the Distribution of dINs}

Our data indicate that Pou2f2 expression is increased in $O C$ deficient dIN populations. To assess whether Pou2f 2 may contribute to the phenotype observed in $\mathrm{Hnf6} / \mathrm{Oc2} 2^{-/-}$ embryos (Kabayiza et al., 2017), we mimicked this increase by overexpressing Pou $2 f 2$ in chicken embryonic spinal cord. Pou $2 f 2$ overexpression did not change the number of dI2 INs, co-labeled for Brn3a and Lhx1/5 (Figures 5A,B). In contrast, it significantly altered their distribution (Figures 5C-E). In HH27-28 control side of the spinal cord (Figure 5C), dI2 cells were distributed in a dorso-medial to ventro-medial manner, with densely packed cells in register with the $\mathrm{dI} 2$ progenitor domain. In contrast, in the electroporated side, the $\mathrm{dI} 2$ distributed in a dorso-medial to ventro-lateral direction with more cells retained dorsally in the densely packed cluster or located in a more lateral position (Figures 5C-E). As for dI2 INs, overexpression of Pou $2 f 2$ in chicken embryonic spinal cord had no effect on the number of dI3 cells (Figures 5F,G). In the control side, dI3 INs distributed in two closely connected clusters in a general dorso-medial to ventro-medial direction (Figure $5 \mathbf{H}$ ). However, in the electroporated side, a majority of dI3 INs remained more dorsal and the population extended laterally (Figures 5H-J). These observations indicate that increased Pou2f 2 did not change $\mathrm{dI} 2$ or $\mathrm{dI} 3$ production but altered the migration of these populations in the dorsal spinal cord. In contrast, overexpression of Pou $2 f 2$ in chicken embryonic spinal cord resulted in a significant increase in $\mathrm{dI} 5 \mathrm{Lmxlb}^{+}$ INs (Figures 5K,L), suggesting that Pou2f2 may promote dI5 production. Regarding their distribution, dI5 Lmx1b ${ }^{+}$ cells were located in the medial region along the DV axis of 

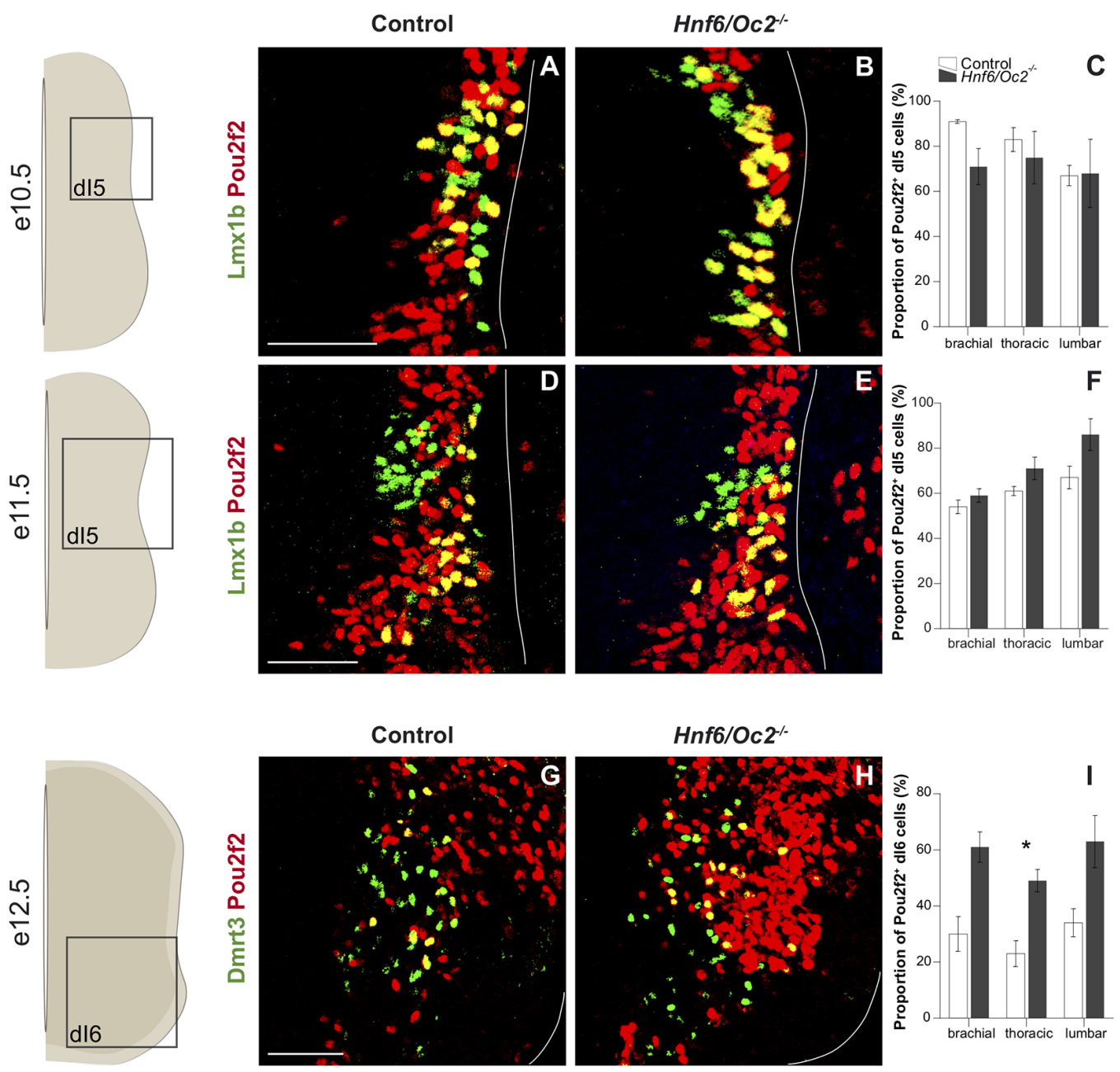

FIGURE 4 | The OC factors moderate Pou2f2 expression in dl5 interneurons and Dmrt3 ${ }^{+}$dl6 interneuron subset. (A-D) Immunodetection of Pou2f2 (red) in $\mathrm{Lm \times 1b^{+ }}$ (green) dl5 neurons on transverse sections of thoracic spinal cord at e10.5 (A,B) and at e11.5 (C,D). Pou2f2 is detected in most of $L m \times 1 b^{+}$ dl5 interneurons at e10.5 and is then restricted to the ventral part of the population. Pou2f2 signal is stronger in $\mathrm{Lm \times 1b^{+ }}$ dl5 interneurons in $\mathrm{Hnf6}_{\mathrm{OCc}} \mathrm{2}^{-/-}$spinal cord at all studied developmental stages. (E,F) The percentage of Pou2f2 positive dl5 neurons was quantified at e10.5 (E) and e11.5 (F), and is not significantly different in Hnf6/Oc2 $2^{-/-}$spinal cords. (G-I) Immunodetection and relative quantification of Pou2f2 in Dmrt3 ${ }^{+}$(green) dl6 subset at e12.5. The proportion of dl6 Dmrt3 ${ }^{+}$ Pou2f2 ${ }^{+}$is significantly increased at thoracic level in Hnf6/Oc2 $2^{-1-}$ mutant embryos $(p \leq 0.05)$. Again, Pou2f2 signal is stronger in the absence of OC factors. The pictures show part of right hemisections as indicated on the schemes to the left. Solid lines delineate the spinal cord. Mean values $\pm \mathrm{SEM}, n=3$. ${ }^{*}=p \leq 0.05$. Scale bars $=100 \mu \mathrm{m}$.

the spinal cord forming a minor medial cluster connected to a major lateral cluster on the medio-lateral axis in the control side of spinal cord (Figures $\mathbf{5} \mathbf{M}, \mathbf{O}$ ). However, this population was more lateral and slightly more ventral in electroporated spinal cord (Figures 5M-O). Finally, given the lack of a specific marker for dI4 and dI6 INs at this early developmental stage, the consequences of increased Pou 2 f expression in the chick spinal cord were studied using Lbx1 and Lhx1/5 as dI4 and dI6 co-markers. As for dI2 and dI3 INs, Pou2f2 increase did not alter the number of dI4:dI6 cells (Figures 5P,Q). In control spinal cords, these cells were located in a dorso-medial to ventromedial pattern. In contrast, in electroporated spinal cords, these INs were distributed in a dorso-medial to ventro-lateral direction with more cells in a lateral position (Figures 5R-T).
Taken together, these data suggest that Pou2f2 is able to modulate the differentiation of dI5 and the distribution of dI2 to dI6 INs in the developing spinal cord. In particular, increased Pou2f 2 resulted in a more dorsal and more lateral distribution of dIN populations.

\section{Pou2f2 Is Not Necessary for Proper Production of dINs}

Our overexpression data suggest that Pou2f 2 is sufficient to modulate dI 5 differentiation and dIN distribution. To determine whether Pou $2 \mathrm{f} 2$ is necessary for these developmental processes, we assessed the differentiation and the distribution of each population of dI2-dI6 INs in Pou2f2 mutant embryos (Corcoran et al., 1993). First, we determined whether early IN production 


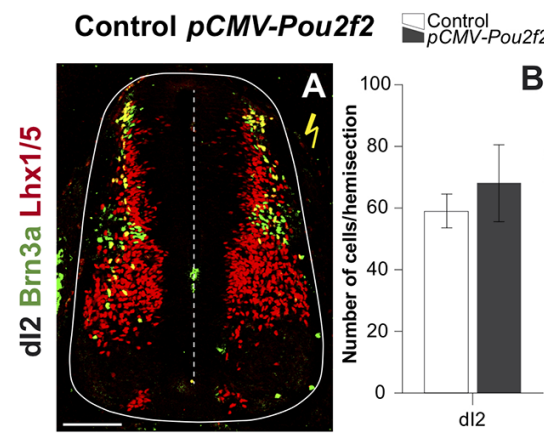

Control
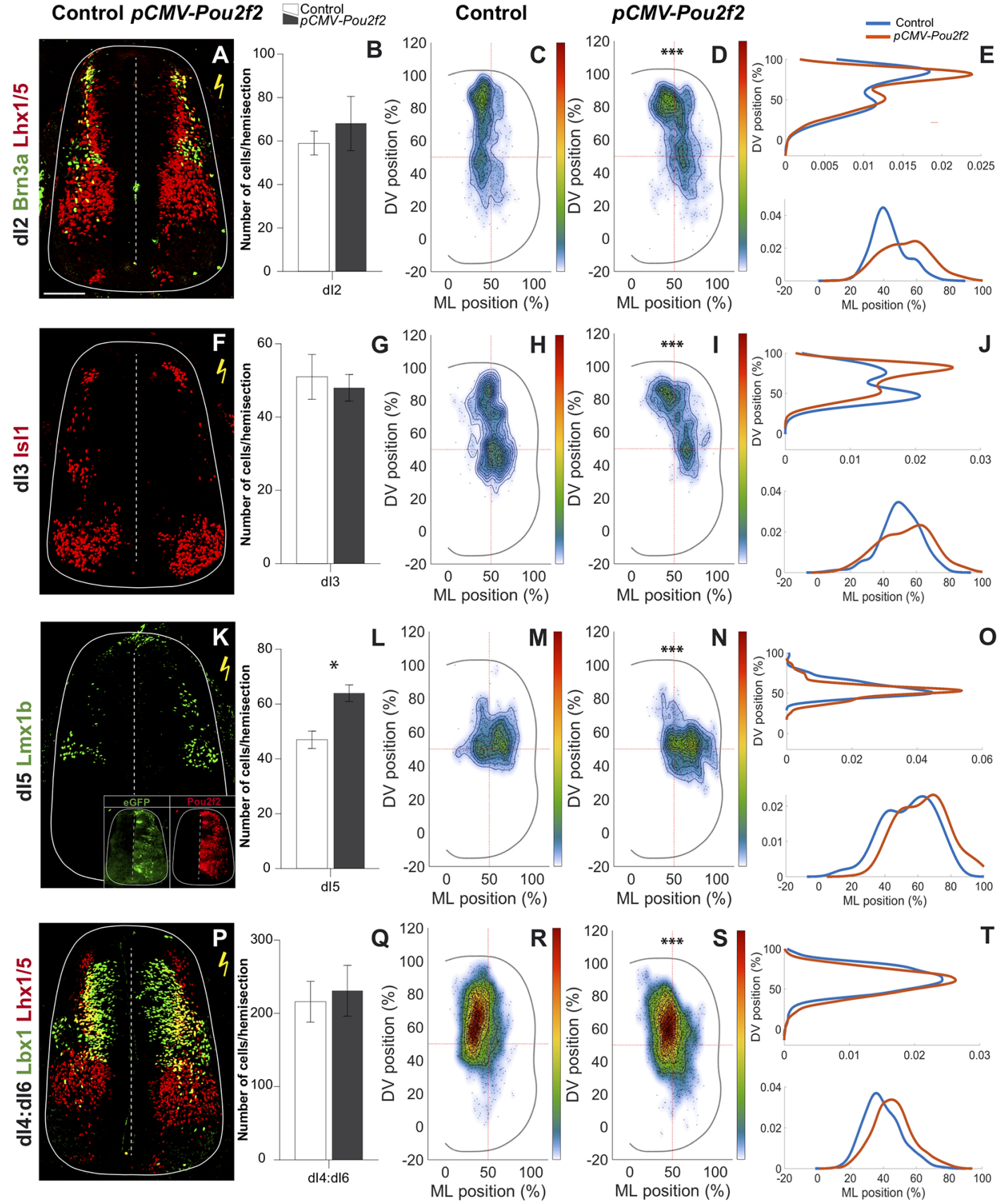

FIGURE 5 | Dorsal interneuron distribution is altered after Pou2f2 overexpression. Overexpression of Pou2f2 in chicken embryonic spinal cord after electroporation at $\mathrm{HH} 14-16$ and immunolabelings $72 \mathrm{~h}$ after electroporation. Insets in (K) show immunodetection of GFP as electroporation control and of Pou2f2 as overexpression control. (A,B) Immunodetection and quantification of $\mathrm{Brn3a}^{+}$(green) Lhx1/5+ (red) dl2 neurons on transverse sections of electroporated spinal cord at $\mathrm{HH} 27-28$. Pou2f2 overexpression does not impact the number of dl2 interneurons. (C-E) Distribution analysis of dl2 interneurons in control or electroporated side of the chicken spinal cord. (C,D) Two-dimension distribution graphs (left) show integration of cell distribution from multiple sections of multiple embryos of each genotype. (E) One-dimension graphs compare density distribution in control (blue) and in electroporated spinal cord (red) on the dorso-ventral (DV; upper) or the medio-lateral (lower) axis of the spinal cord (see "Materials and Methods" section for details). Overexpression of Pou2f2 alters the distribution of dl2 cells. (C) In control spinal cord, dl2 interneurons are distributed in a dorso-medial to ventro-medial fashion, with densely packed neurons close to the dl2 progenitor domain. (D,E) In electroporated spinal cord, dl2 cells migrate in a dorso-medial to ventro-lateral direction with more cells retained dorsally or located in a more lateral position $(p \leq 0.001)$.

$\mathbf{( F , G ) ~ I m m u n o d e t e c t i o n ~ a n d ~ q u a n t i f i c a t i o n ~ o f ~} \mathrm{ISI} 1^{+}$(red) dl3 neurons on transverse sections of electroporated spinal cord at $\mathrm{HH} 27-28$. All the Is $11^{+}$cells dorsal to the motor columns are considered as dl3. The number of dl3 interneurons is not modified after Pou2f2 overexpression. (H) In control spinal cord, dl3 are distributed in two closely connected clusters in a dorso-medial to ventro-medial direction. $(\mathbf{I}, \mathbf{J})$ In electroporated spinal cord, the dl3 interneurons are more dorsal and their lateral migration is increased $(p \leq 0.001)$. (K,L) Immunodetection and quantification of $\mathrm{Lmx} 1 \mathrm{~b}^{+}$(green) dl5 neurons on transverse sections of electroporated spinal cord at 
FIGURE 5 | Continued

$\mathrm{HH} 27-28$. The number of dl5 interneurons is significantly increased after Pou2f2 overexpression. Moreover, their distribution is altered. (M) In control spinal cord, dl5 are distributed in two connected minor and major clusters both located in the medial tier of the spinal cord. $(\mathbf{N}, \mathbf{O})$ In electroporated spinal cord, the dl5 are located more laterally and slightly more ventrally $(p \leq 0.001)$. $(\mathbf{P}, \mathbf{Q})$ Immunodetection and quantification of $\mathrm{Lbx} 1^{+}$(green) Lhx $1 / 5^{+}$(red) dl4:dl6 neurons on transverse sections of electroporated spinal cord at HH27-28. Pou2f2 overexpression does not alter the number of dl4 + dl6 neurons. (R) In control spinal cord, dl $4+$ dl 6 are distributed in a dorso-medial to ventro-medial pattern. (S,T) In electroporated spinal cord, the dl $4+$ dl6 neurons organize in a dorso-medial to ventro-lateral fashion with more cells in a lateral position. Mean values $\pm \mathrm{SEM}, n=3$, five sections per embryo $\left(n>710\right.$ cells per condition). ${ }^{* * *}=p \leq 0.001$. Scale bar $=100 \mu \mathrm{m}$.

was normal at e10.5 in the absence of Pou2f2. The number of dI 2 cells, labeled for Foxd3, was similar in control and in Pou2f2 mutants at each level of the spinal cord, as confirmed by cell quantification (Figures 6A-C), although the early distribution of these cells seemed different (Figures 6A,B). Similar analyses were carried out for dI3-dI6 populations. All those populations were produced in normal numbers in Pou $2 f^{-/-}$embryos compared to control littermates (Figures 6D-L). Taken together, these results suggest that Pou2f 2 is not necessary for the early steps of dIN differentiation.

\section{Pou2f2 Controls the Distribution of dl2 INs}

However, the early migration of some dIN populations seemed affected at e10.5 (Figure 6). Given that OC factors regulate the distribution of dINs in the embryonic spinal cord (Kabayiza et al., 2017), that OC proteins repress Pou $2 f 2$ expression in spinal INs (Figures 1-4 and Harris et al., 2019) and that Pou2f2 is able to modulate the position of these cells (Figure 5), we assessed whether the loss of Pou $2 f 2$ impacts on dIN distribution at e12.5 and e14.5, i.e., in the course of interneuron migration and when migration of these cells in the transverse plan of the spinal cord is completed, respectively. To discriminate dI2 INs located in ventral regions from V1 cells, which also produce Foxd3, dI2 were additionally labeled for Brn3a. As observed at e10.5, the number of dI2 INs was not altered at e12.5 in the absence of Pou2f2 (Figures 7A-C). In control embryos, a majority of dI 2 cells distributed in a medial stream of cells migrating from the dI2 progenitor domain towards the ventral region of the spinal cord and covering $60 \%$ of the ventro-dorsal axis. A second cluster of dI2 neurons expressing high amounts of Brn3a (arrow in Figure 7A) was located ventrally in the vicinity of the Foxd $3^{+}$ V1 INs (Figures 7D-F). In Pou $2 f 2^{-/-}$mutant embryos, cells producing Foxd3 and Brn3a were detected in similar regions (Figure 7B). However, distribution analysis showed that dI2 INs migrate more ventrally in the absence of Pou2f2, connecting the dorsal and ventral clusters at brachial level and increasing the number of cells in the ventral cluster at thoracic and lumbar levels (Figures 7G-L).

At e14.5, the number of dI2 cells at brachial level was mildly but significantly increased in Pou $22^{-/-}$mutant embryos (Figures 7M-O). In control embryos, a main dI2 cluster settled in the intermediate part of the medial spinal cord (arrow in Figures $\mathbf{7 M}, \mathbf{P}-\mathbf{R}$ ) whereas two smaller subsets located in a more ventral or lateral position, respectively (arrowheads in Figures $\mathbf{7 M}, \mathbf{P}-\mathbf{R}$ ). In mutant embryos, the cells distributed in a similar pattern to control littermates although, reminiscent of e12.5, dI2 were located more ventrally and the small lateral cluster was depleted at thoracic and lumbar levels (Figures 7N,S-X). Taken together, these observations suggest that Pou2f2 regulates the distribution of dI2 INs.

\section{Pou2f2 Controls the Distribution of dl3 INs}

Except for a mild but significant reduction at e12.5 at brachial level, the number of $\mathrm{dI} 3$ INs characterized by the expression of Isl1 was not affected in the absence of Pou $2 f 2$ (Figures 8A-C,M-O). At e12.5, the dI 3 cells gathered as a single homogeneous cluster in the intermediate region of the spinal cord, resulting in a Gaussian-like distribution along the DV and the medio-lateral axes (Figures 8A,D-F). In Pou $2 f 2^{-/-}$spinal cords, the population was slightly more ventral at brachial level and more lateral at thoracic and lumbar levels, respectively, with a more diffuse distribution in the center of the cluster (Figures $\mathbf{8 B}, \mathbf{G}-\mathbf{L}$ ). Two days later, still gathered in a single cluster, dI3 neurons settled in the intermediate region of the spinal cord. The dI3 INs located more ventral and lateral in the absence of Pou2f2 (Figures $\mathbf{8 P}-\mathbf{X}$ ). In addition, a tiny ectopic dI3 cluster was detected at brachial level, whereas a similarly located cluster was missing at lumbar level (arrowheads in Figures 8S,U). These observations suggest, as observed for $\mathrm{dI} 2$ cells, that Pou $2 \mathrm{f} 2$ controls the distribution of $\mathrm{dI} 3 \mathrm{INs}$ in the developing spinal cord.

\section{Pou2f2 Controls the Differentiation and the Distribution of dI5 INs}

In the mouse developing spinal cord, from e11.0 onwards, Lmx1b is present in the dI5 and in the late born $\mathrm{dIL}^{\mathrm{B}}$ INs. As earlyand late-born $\mathrm{Lmxlb}^{+}$cells are difficult to discriminate for reproducible distribution studies, we restricted our analyses to the Phox $2 \mathrm{a}^{+}$dI5 subset at e12.5 and e14.5 (Figure 9). Absence of Pou $2 \mathrm{f} 2$ did not impact the number of dI5 Phox $2 \mathrm{a}^{+}$INs except for the lumbar level at e14.5, which showed a significant increase (Figures 9A-C,M-O). In control embryos at e12.5, Phox $2 \mathrm{a}^{+}$ dI5 distributed in 2 main clusters on the medio-lateral axis of the spinal cord, with a major medial cluster and a minor lateral cluster that trended to coalesce in more caudal sections (Figures 9A,D-F). In Pou $22^{-/-}$embryos, dI5 distribution was dramatically changed with a majority of cells organized in a unique cluster located in a medial position (Figures $\mathbf{9 B}, \mathbf{G}-\mathbf{L}$ ). At e14.5, control Phox $2 \mathrm{a}^{+}$dI5 gathered in a main medial cluster located more dorsally in more caudal sections (Figures $\mathbf{9 M}, \mathbf{P}-\mathbf{R}$ ). In mutant embryos, cells were more compact but occupied a more lateral position at brachial and thoracic levels, and a more dorsal position at lumber level (Figures 9N,S-X). These observations indicate that Pou $2 \mathrm{f} 2$ regulates the differentiation and the distribution of the Phox $2 \mathrm{a}^{+}$dI5 subpopulation.

\section{Pou2f2 Controls the Distribution of dl6 INs}

At e12.5 and e14.5, two partially overlapping dI6 subpopulations are characterized by the presence of Dmrt3 or WT1, respectively. Absence of Pou 22 had no impact on 


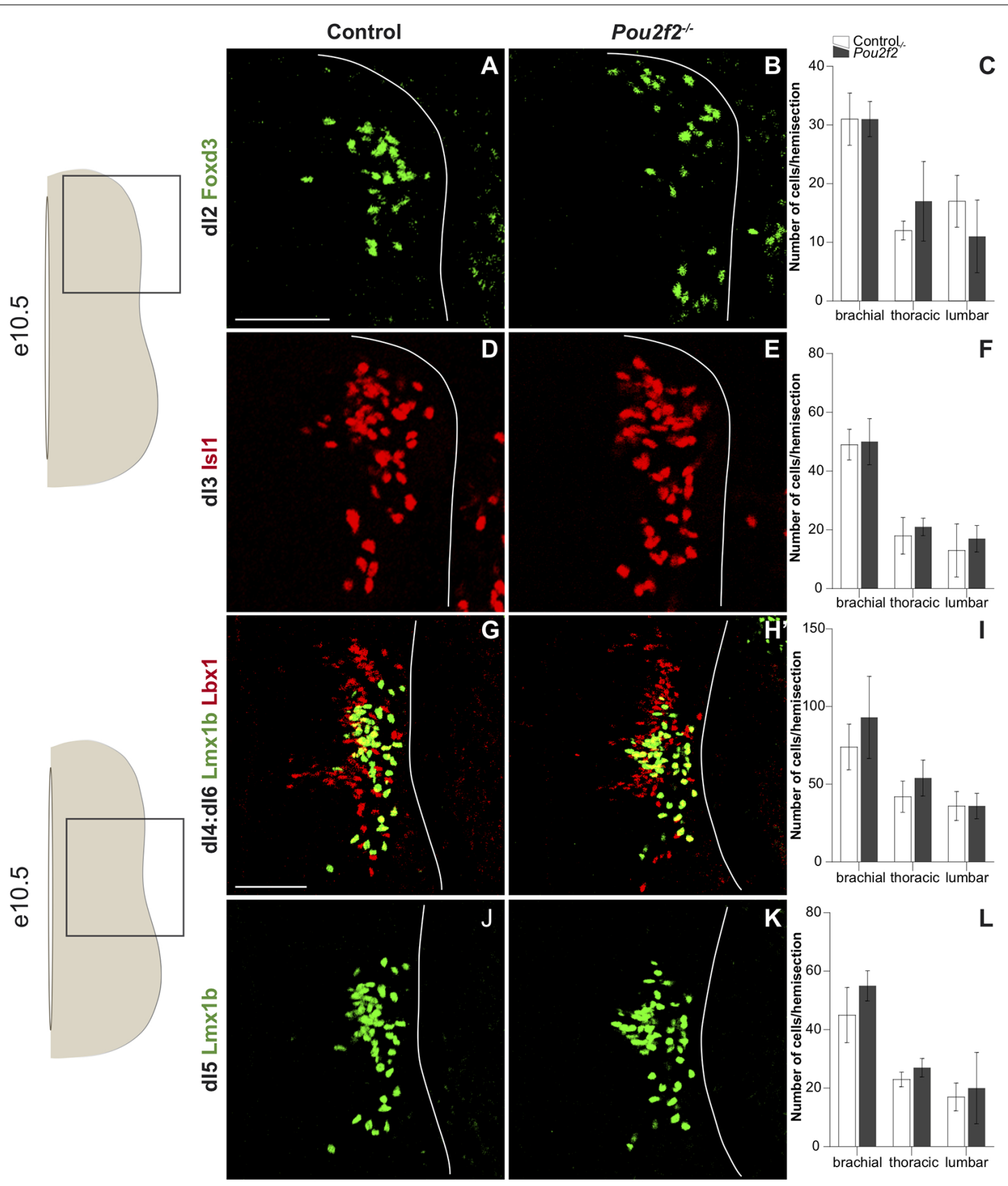

FIGURE 6 | Dorsal interneuron production is normal at e10.5 in Pou2f2 mutant embryos. (A-L) Immunodetection and quantification of Foxd3 ${ }^{+}$(green) dl2 neurons (A-C), Isl1+ (red) dl3 neurons (D-F), Lmx1b+ (green) Lbx1+ (red) dl4:dl6 neurons (G-I) and Lmx1b+ (green) dl5 neurons (J-L) on transverse sections of thoracic spinal cord at e10.5. Quantifications show comparable numbers of dl2 (C), dl3 (F), dl4:dl6 (I) and dl5 (L) cells in control or Pou2f2 ${ }^{-/-}$mutant embryos, indicating that the production of these populations is not affected in the absence of Pou2f2. The pictures show part of right hemisections as indicated on the schemes to the left. Solid lines delineate the spinal cord. Mean values \pm SEM, $n=3$. Scale bar $=100 \mu \mathrm{m}$.

dI6 cell number of each subpopulation but resulted in alterations in $\mathrm{Dmrt}^{+}$(Figures 10A-C) and in $\mathrm{WT}^{+}$ (Figures 11A-C) dI6 distribution. At e12.5, control Dmrt3 ${ }^{+}$ dI6 INs were located in the ventro-medial part of the spinal cord (Figures 10A,D-F). In Pou $22^{-/-}$embryos, $\mathrm{Dmrt3}^{+}$INs settled more ventrally at brachial level than in mutant embryos (Figures 10B,G-L). Due to technical restrictions, we limited our distribution analysis of $\mathrm{Dmrt}^{+}$ dI6 subset to e12.5.

In control embryos at e12.5, the $\mathrm{WT}^{+} \mathrm{dI} 6$ subset was located, similarly to the Dmrt $3^{+}$subpopulation, in the ventro-medial part of the spinal cord (Figures 11A,D-F). In Pou $2 f 2^{-/-}$embryos, WT1 ${ }^{+}$dI6 INs were more densely packed and were located more ventrally than in control 


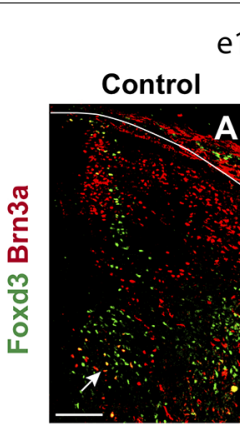

e12.5

\section{e14.5}
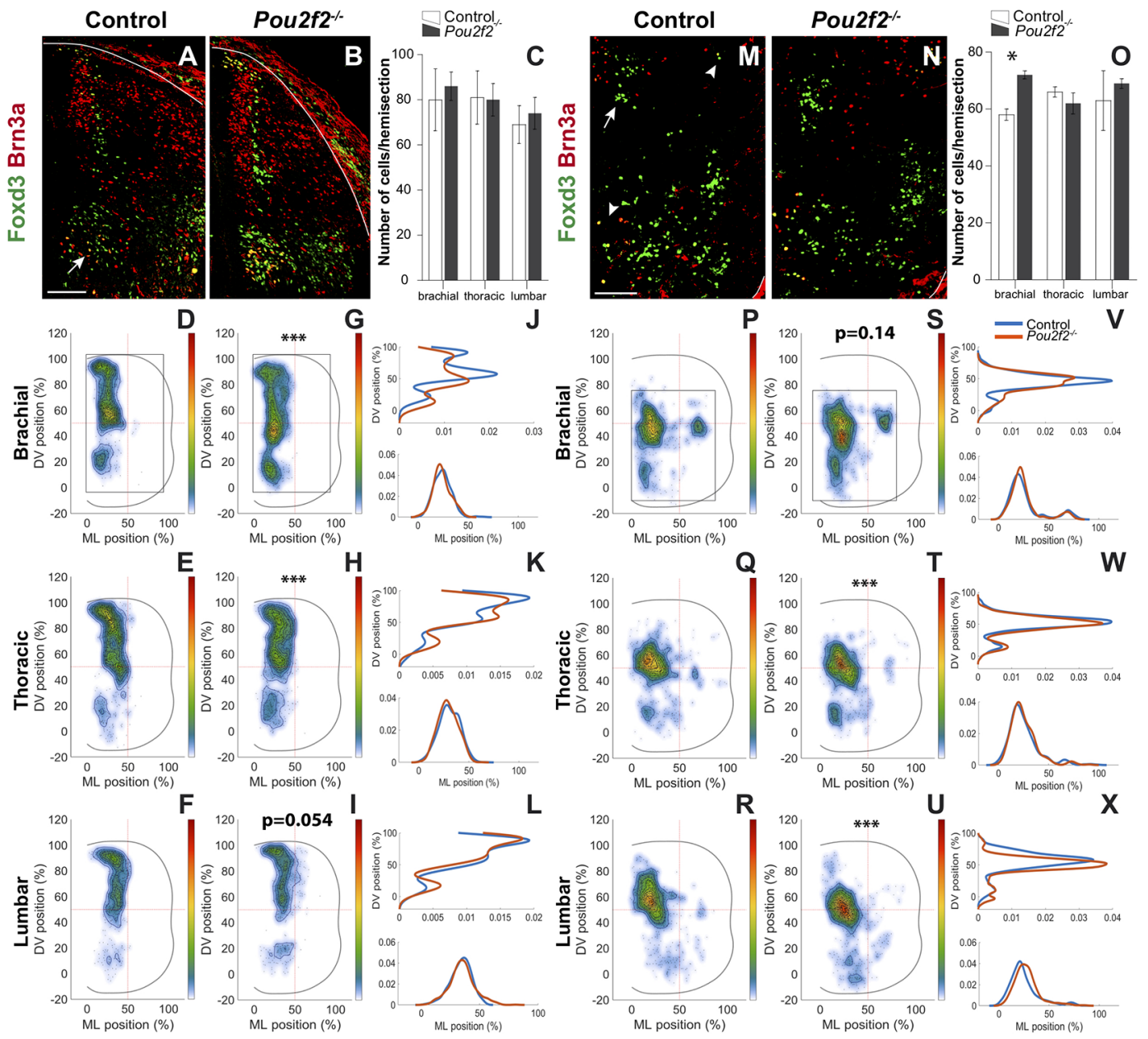

FIGURE 7 | Pou2f2 regulates the distribution of dl2 interneurons. (A-C) Immunodetection and quantification of Foxd3 ${ }^{+}$(green) Brn3a+ (red) dl2 interneurons in control or Pou2f2-/- mutant embryos at e12.5. The production of the Foxd3 ${ }^{+}$Brn3a $^{+}$dl2 interneurons is not altered in the absence of Pou2f2. (D-L) Distribution of dl2 interneurons on the transverse plane of the spinal cord in control or Pou2f2-/- mutant embryos at e12.5. Two-dimension distribution graphs (left) show integration of cell distribution from multiple sections of multiple embryos of each genotype. One-dimension graphs (right) show density distribution in control (blue) or in Pou2f2 $2^{-/-}$embryos (red) on the DV (upper) or the medio-lateral (lower) axis of the spinal cord. The distribution of dl2 interneurons is affected in Pou2f2 ${ }^{-/-}$ mutants, as dl2 cells migrate more ventrally, connecting the dorsal and ventral clusters at brachial level $(p \leq 0.001)$ and increasing the number of cells in the ventral cluster at thoracic $(p \leq 0.001)$ but not at lumbar levels $(p=0.0536)$. (M-0) Immunodetection and quantification of Foxd3 ${ }^{+}$(green) Brn3a ${ }^{+}$(red) dl2 interneurons in control or Pou2f2-l- mutant embryos at e14.5. The number of Foxd3 ${ }^{+}$Brn3a $^{+} \mathrm{dl} 2$ cells is increased at the brachial level, but not at more dorsal levels, in the absence of Pou2f2 ( $p \leq 0.05)$. (P-X) Distribution of dl2 interneurons on the transverse plane of the spinal cord in control or Pou2f2 ${ }^{-/-}$mutant embryos at e14.5. In the absence of Pou2f2, dl2 neurons settle more ventrally and small lateral clusters are depleted at thoracic and lumbar levels ( $p \leq 0.001)$. The pictures show part of right hemisections as indicated on the schemes in (D, G) and in (P,S), respectively. Solid lines delineate the spinal cord. Mean values \pm SEM, $n=3$, five sections per level for each embryo ( $n>2,803$ cells per condition). ${ }^{*}=p \leq 0.05 ;{ }^{* * *}=p \leq 0.001$. Scale bar $=100 \mu \mathrm{m}$.

littermates (Figures 11B,G-L). Consistently, at e14.5, WT1 ${ }^{+}$ cells remained more densely packed and more ventral than in control littermates (Figures $\mathbf{1 1} \mathbf{M}-\mathbf{X}$ ). These observations indicate that Pou $2 \mathrm{f} 2$ controls some aspects of dI6 distribution in the developing spinal cord.

\section{DISCUSSION}

During spinal cord development, proper cell migration is critically required for adequate integration of post-mitotic neurons into specific neural circuits. Recent studies demonstrated the importance of correct population and subpopulation distribution for spinal circuitry formation (Sürmeli et al., 2011; Tripodi et al., 2011; Goetz et al., 2015; Hinckley et al., 2015; Lu et al., 2015; Bikoff et al., 2016; Hilde et al., 2016). Although the involvement of an extensive amount of guidance molecules in the regulation of neuronal migration has been well characterized (Chen, 2019), the genetic programs that control the production of these guidance molecules and the selective responsiveness of distinct neuronal populations to these cues are still poorly understood, particularly in the spinal cord. Here we provide evidence that a genetic cascade composed 


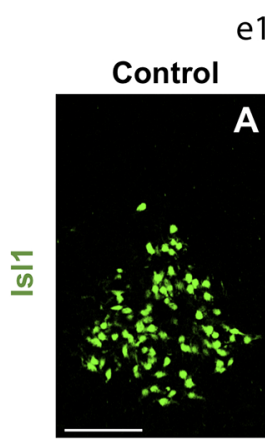

$\mathrm{e} 12.5$

Pou2f2-
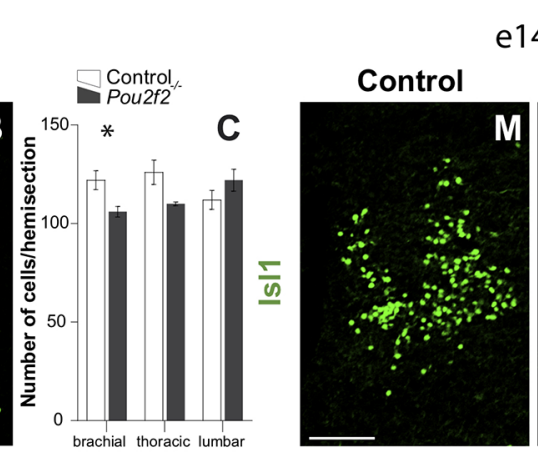

e14.5
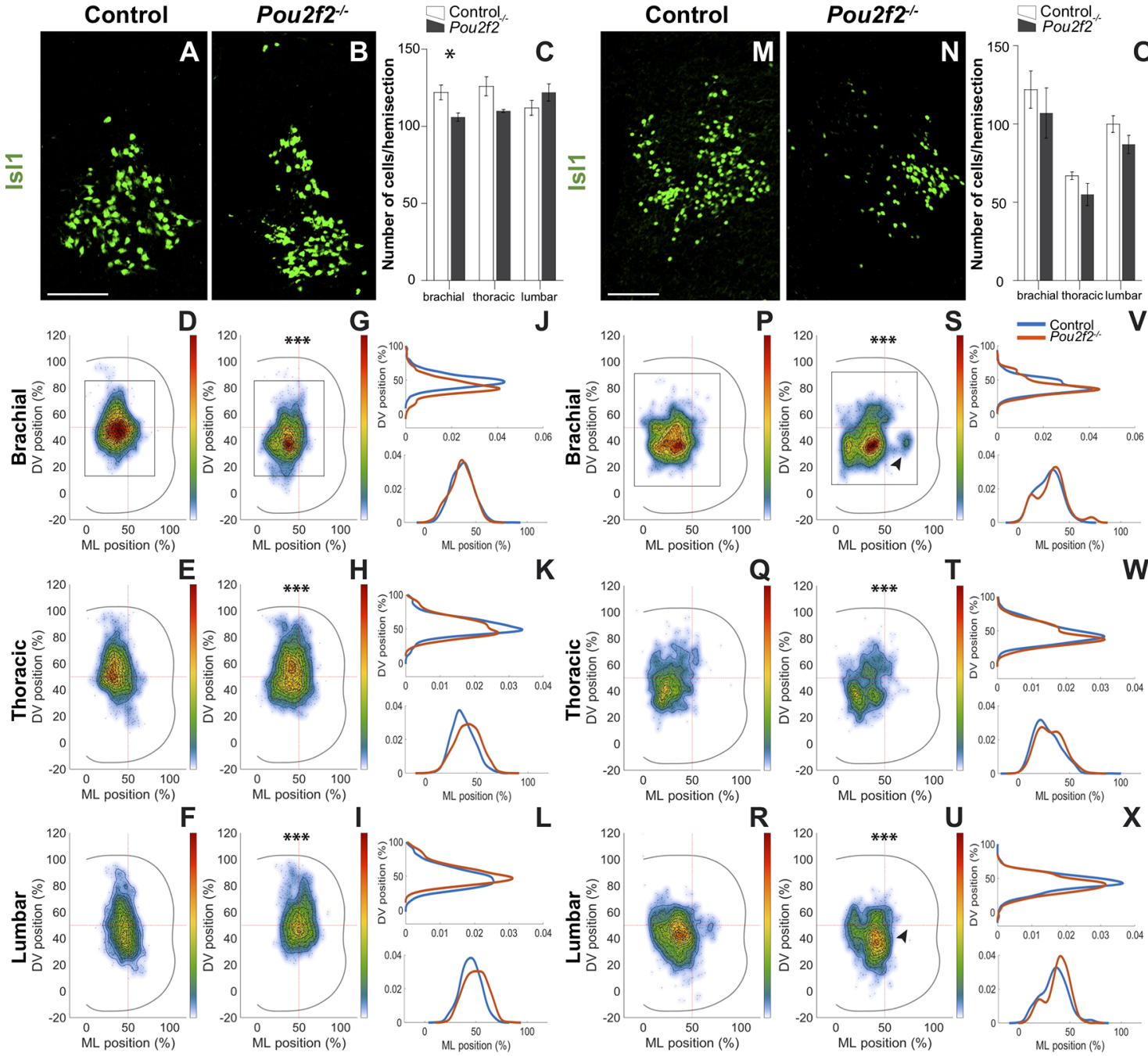

W

FIGURE 8 | Pou2f2 regulates the distribution of dl3 interneurons. (A-C) Immunodetection and quantification of Isl1 ${ }^{+}$(green) dl3 interneurons in control or Pou2f2 ${ }^{-/-}$mutant embryos at e12.5. The production of the Isl1+ $\mathrm{dl3}$ interneurons is not altered in the absence of Pou2f2 except for a slight decrease at brachial level $(p \leq 0.05)$. (D-L) Distribution of dl3 interneurons on the transverse plane of the spinal cord in control or Pou2f2 ${ }^{-1-}$ mutant embryos. Two-dimension distribution graphs (left) show integration of cell distribution from multiple sections of multiple embryos of each genotype. One-dimension graphs (right) show density distribution in control (blue) or in Pou2f2-/- embryos (red) on the DV (upper) or the medio-lateral (lower) axis of the spinal cord. In control spinal cord, dl3 are distributed as a single homogeneous cluster. In Pou2f2-/- mutant embryos, dl3 cells are relatively more ventral at brachial level or lateral at thoracic and lumbar levels ( $p \leq 0.001)$. (M-O) Immunodetection and quantification of Isl1 ${ }^{+}$(green) dl3 interneurons in control or Pou2f2 ${ }^{-/-}$mutant embryos at e14.5. The number of Isl1 ${ }^{+}$dl3 cells is unaffected in the absence of Pou2f2. (P-X) Distribution of dl3 interneurons on the transverse plane of the spinal cord in control or Pou2f2 ${ }^{-/-}$mutant embryos at e14.5. Still organized in a single cluster, dl3 in control embryos settle in the intermediate to ventral regions of the spinal cord. More ventral and lateral distribution is detected in Pou2f2 depleted embryos $(p \leq 0.001)$. A tiny ectopic cluster is present at brachial level or absent at lumbar level. The pictures show part of right hemisections as indicated on the schemes in $\mathbf{(} \mathbf{D}, \mathbf{G})$ and in $\mathbf{( P , S )}$, respectively. Mean values $\pm \mathbf{S E M}, n=3$, five sections per level for each embryo $(n>3,724$ cells per condition). ${ }^{*}=p \leq 0.05 ;{ }^{* * *}=p \leq 0.001$. Scale bar $=100 \mu \mathrm{m}$.

of OC factors and their downstream target Pou 2 f2 controls the distribution of dINs in the developing spinal cord.

\section{OC Factors Repress Pou2f2 Expression in the Developing Spinal Cord}

The diversification and the distribution of dIN have been recently shown to be regulated by OC transcription factors (Kabayiza et al., 2017). In an effort to identify downstream effectors involved in this process, we uncovered genes regulated by OC factors in the embryonic spinal cord. Only a limited number of genes coding for guidance cues were identified. Moreover, none of the candidate genes known to control neuronal migration were confirmed to be under OC protein regulation in the spinal cord. The outcome of this analysis suggests that the migration of each spinal neuronal population may be regulated by diverse micro-environmental cues and receptors, instead of 

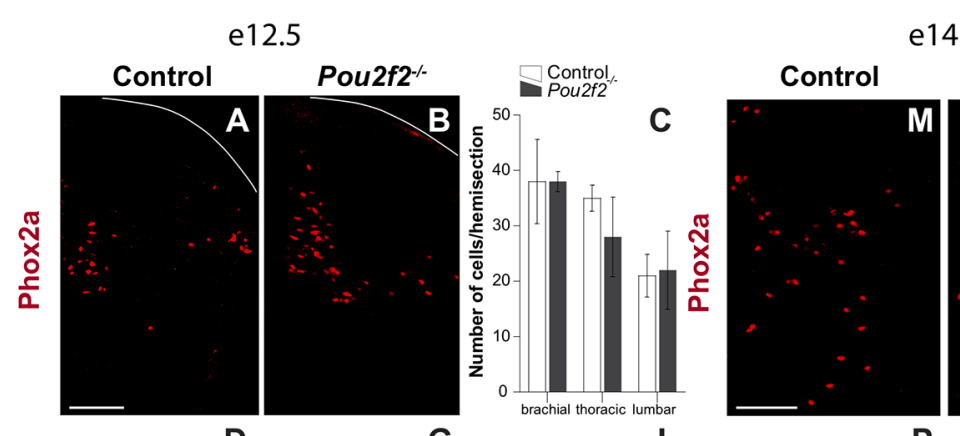

e14.5
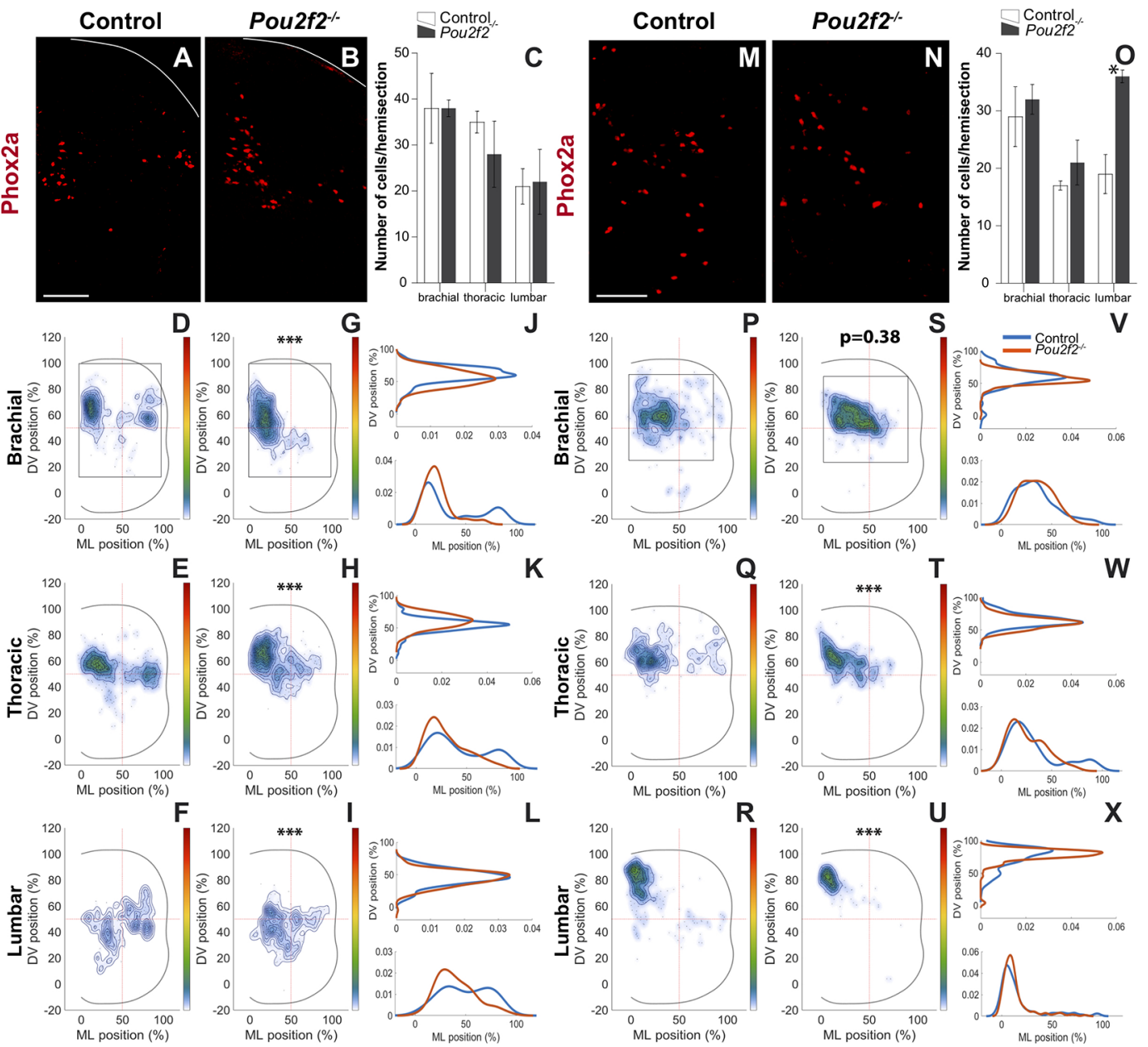

FIGURE 9 | Pou2f2 regulates the distribution of dl5 Phox2a subset. (A-C) Immunodetection and quantification of dl5 Phox2a ${ }^{+}$(red) subset in control or Pou2f2-/mutant embryos at e12.5. The production of the Phox $2 \mathrm{a}^{+}$dl5 interneurons is not altered in the absence of Pou2f2. (D-L) Distribution of dl5 Phox2a ${ }^{+}$cells on the transverse plane of the spinal cord in control or Pou2f2 ${ }^{-/-}$mutant embryos. Two-dimension distribution graphs (left) show integration of cell distribution from multiple sections of multiple embryos of each genotype. One-dimension graphs (right) show density distribution in control (blue) or in Pou2f2-/- embryos (red) on the DV (upper) or the medio-lateral (lower) axis of the spinal cord. The distribution of dl5 Phox2a+ subset is dramatically affected in Pou2f2-/- mutants. In control embryos, dl5 $\mathrm{Phox}_{2} \mathrm{a}^{+}$cells are organized in a major medial and a minor lateral cluster that progressively coalesce in more caudal regions. In Pou2f2-deficient spinal cords, a unique cluster located in a medial position is observed $(p \leq 0.001)$. (M-0) Immunodetection and quantification of dl5 Phox2a ${ }^{+}$(red) subset in control or Pou2f2 ${ }^{-/-}$ mutant embryos at e14.5. The number of Phox $2 \mathrm{a}^{+}$dl5 cells is significantly increased at lumbar level $(p \leq 0.05)$. (P-X) Distribution of dl5 Phox2a ${ }^{+}$neurons on the transverse plane of the spinal cord in control or Pou2f2-/- mutant embryos at e14.5. In control embryos, dl5 Phox2a+ gathered in a main medial cluster whereas, in Pou2f2-/- spinal cords, cells were organized in a more compact cluster shifted laterally at brachial and thoracic levels and more dorsally at lumbar level ( $p \leq 0.001)$. The pictures show part of right hemisections as indicated on the schemes in $\mathbf{( D , G )}$ and in $\mathbf{( P , S )}$, respectively. Solid lines delineate the spinal cord. Mean values $\pm \mathrm{SEM}, n=3$, five sections per level for each embryo $\left(n>930\right.$ cells per condition). ${ }^{*}=p \leq 0.05 ; * * *=p \leq 0.001$. Scale bar $=100 \mu \mathrm{m}$.

a generic mechanism common to all populations. The diversity of migration routes and endpoints (Lu et al., 2015; Lai et al., 2016; Chen, 2019) is consistent with this hypothesis. However, expression of the Pou2f2 transcription factor appeared to be repressed by the OC factors in the embryonic spinal cord. OC inactivation did not result in a significant increase in the number of dINs containing Pou2f2, unless for the dI6 Dmrt3 ${ }^{+}$subset. In contrast, cells expressing Pou 2 2 in control embryos show increased Pou 22 levels in $O C$ mutant spinal cord. Thus, OC factors seem to moderate Pou $2 f 2$ expression in its endogenous domain rather than preventing an ectopic activation in other
dINs subpopulations. However, OC factors are supposed to mainly behave as transcriptional activators (Jacquemin et al., 2000, 2003; Pierreux et al., 2004; Roy et al., 2012). Therefore, our observations suggest an indirect regulation of Pou $2 f 2$ expression by the OC factors. Alternatively, we can not exclude that $\mathrm{OC}$ factors may exert a dual role as both a transcriptional activator or a repressor depending on the cell type, as previously shown for Gli3, which can act both as an activator or as a repressor during different phases of zebrafish CNS patterning (Tyurina et al., 2005), or Arx known for its bifunctional activity during Xenopus forebrain development (Seufert et al., 2005). Identification of 


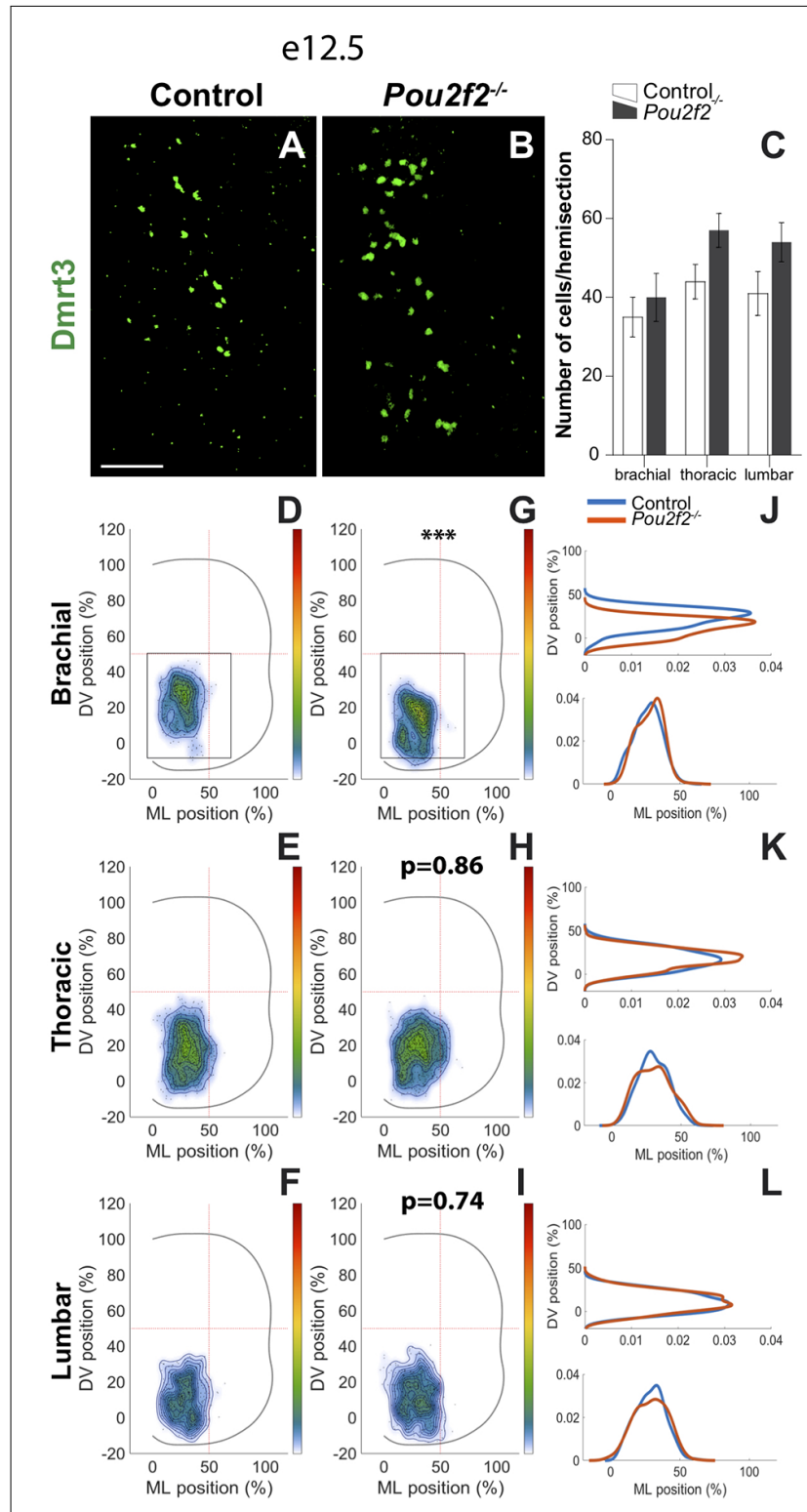

FIGURE 10 | Pou2f2 regulates the distribution of the dl6 Dmrt3 subset. (A-C) Immunodetection and quantification of dl6 interneuron Dmrt3 ${ }^{+}$(green) subset in control or Pou2f2 ${ }^{-/-}$mutant embryos at e12.5. The production of the Dmrt3 ${ }^{+}$dl6 interneurons is not altered in the absence of Pou2f2. (D-L) Distribution of dl6 Dmrt3 ${ }^{+}$cells on the transverse plane of the spinal cord in control or Pou2f2 $2^{-/-}$mutant embryos. Two-dimension distribution graphs (left) show integration of cell distribution from multiple sections of multiple embryos of each genotype. One-dimension graphs (right) show density distribution in control (blue) or in Pou2f2 ${ }^{-/-}$embryos (red) on the DV (upper) or the medio-lateral (lower) axis of the spinal cord. Dmrt3 ${ }^{+}$dl6 distribution is affected in Pou2f2-l- mutants at brachial level as these cells settle more ventrally relatively to control littermates $(p \leq 0.001)$. The pictures show part of right hemisections as indicated on the schemes in $\mathbf{( D , G ) . ~ M e a n ~}$ values \pm SEM, $n=3$, five sections per level for each embryo $(n>1,700$ cells per condition). ${ }^{* * *}=p \leq 0.001$. Scale bar $=100 \mu \mathrm{m}$.

the $5^{\prime}$ sequences of the embryonic spinal Pou $f 2$ transcripts (Harris et al., 2019) and of the regulating sequence controlling the expression of Pou $2 \mathrm{f} 2$ in the developing spinal cord would be required to unveil the mechanisms whereby OC regulate Pou $2 f 2$ expression. Nevertheless, our analysis uncovered Pou $2 f 2$ as a downstream target of OC factors in the dINs.

\section{An OC-Pou2f2 Genetic Cascade Regulates the Distribution of dINs}

To assess whether Pou2f 2 may regulate dIN distribution, we increased Pou $2 f 2$ expression in the spinal cord using chicken embryonic electroporation. Pou $2 f 2$ overexpression resulted in dINs mislocalization without any change in their cell number, except for the dI5 Phox2a subset. These alterations are reminiscent of dIN alterations observed in the absence of OC factors (Kabayiza et al., 2017), even if in ovo electroporation constrains the analysis to developmental stages earlier than those studied in the mouse, which hinders the evaluation of terminal settling position of the dINs. In $H n f 6 / O c 2^{-/-}$embryos, dI3 INs displayed altered migration trajectory (Kabayiza et al., 2017). Similarly, after Pou $2 f 2$ overexpression, chicken dI3 migration pathway was altered. Furthermore, the dI5 INs were mislocalized after Pou $2 f 2$ overexpression, as observed for the Phox2a-positive dI5 subset in $\mathrm{Hnf6/Oc2^{-/- }}$ embryos. However, these changes in distribution were not strictly identical, likely owing to differences in the timing, the spatial extent and the level of Pou $2 f 2$ overexpression between the mouse and the chicken models.

Consistently, Pou2f2 inactivation affected dIN distribution with only mild effects on the size of each population. Different types of localization defects were observed in Pou $2 f 2^{-/-}$ embryos. First, the ventral part of dI2 INs, dI6 Dmrt3 and dI6 Wt1 INs subsets settled in more ventral localizations. These cells seemed to be more advanced in their migration process, reaching faster and even going beyond their targeted localization. Second, dI5 Phox 2 a subset showed the most dramatic distribution defect, as its organization was altered from two clusters to a unique main cluster. Variations in these localization defects suggest, as proposed above, that different migration cues are affected in each dIN population. Nevertheless, these observations demonstrate that Pou2f2 contributes to regulating dIN migration. Furthermore, a comparison of distribution alterations between Pou $2 f 2$ and $O C$ mutant embryos points to a possible contribution of Pou $2 \mathrm{f} 2$ downstream of OC factors in the control of dIN migration. Indeed, Pou $2 f 2$ depletion resulted in a relatively loose $\mathrm{dI} 3$ population, whereas dI3 were more compactly distributed in OC mutants. Inversely, Phox2a-positive dI5 were more densely packed along the DV axis in Pou2f2 mutants but more spread in $O C$ mutants. However, although depletion or overexpression of Pou $2 f 2$ might have opposite effects on the expression of its downstream guidance cue targets, these will not necessarily lead to opposite effects on cellular migration. Nevertheless, we propose that a genetic cascade involving $\mathrm{OC}$ and Pou2f2 transcription factors controls the distribution of dI2-dI6 INs in the developing spinal cord.

\section{Pou2f2 Acts as a Regulator of dIN Distribution}

Although Pou $2 \mathrm{f} 2$ has been previously described as a transcription factor regulating the maturation of $\mathrm{B}$ cell precursors into 


\section{e12.5}
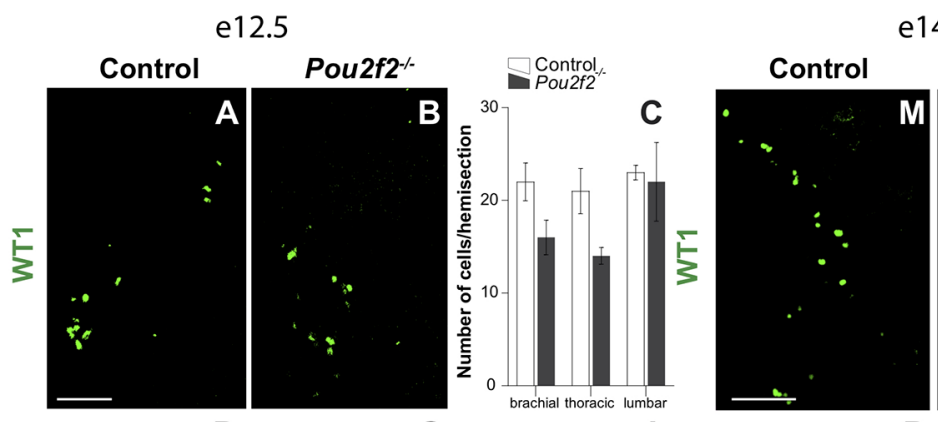

e14.5
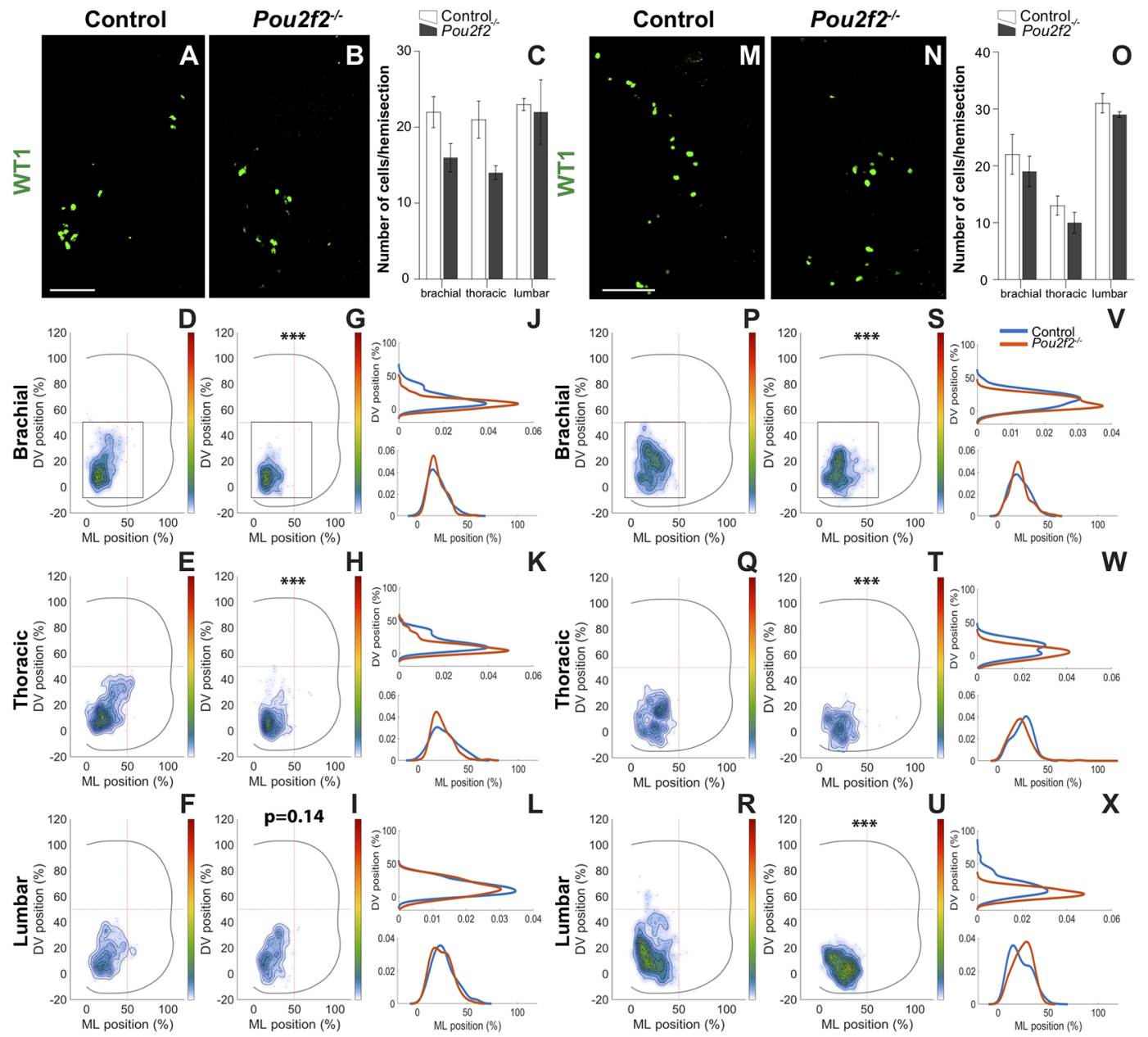

FIGURE 11 | Pou2f2 regulates the distribution of dl6 Wt1 subset. (A-C) Immunodetection and quantification of dl6 Wt1 ${ }^{+}$(green) subset in control or Pou2f2-/mutant embryos at e12.5. The production of the $\mathrm{Wt}{ }^{+}$dl6 interneurons is not altered in the absence of Pou2f2. (D-L) Distribution of dl6 Wt $1^{+}$neurons on the transverse plane of the spinal cord in control or Pou2f2 ${ }^{-/-}$mutant embryos. Two-dimension distribution graphs (left) show integration of cell distribution from multiple sections of multiple embryos of each genotype. One-dimension graphs (right) show density distribution in control (blue) or in Pou2f2 ${ }^{-/-}$embryos (red) on the dorso-ventral (upper) or the medio-lateral (lower) axis of the spinal cord. In control spinal cord, the dl $6 \mathrm{Wt} 1^{+}$subset is localized in the ventro-medial tier. In Pou2f2-/mutants, at brachial and thoracic levels, cells were more densely clustered and located more ventrally and medially ( $p \leq 0.001)$. (M-0) Immunodetection and quantification of $\mathrm{dl}_{6} \mathrm{Wt}^{+}{ }^{+}$(green) subset in control or Pou2f2-/- mutant embryos at e14.5. The size of the Wt1 ${ }^{+} \mathrm{dl} 6$ subset is not altered in the absence of Pou2f2. (P-X) Distribution of dl6 Wt1 ${ }^{+}$cells on the transverse plane of the spinal cord in control or Pou2f2-/- mutant embryos at e14.5. Reminiscent of e12.5, dl6 Wt1 ${ }^{+}$ subset remained more ventrally and more densely packed in the absence of Pou2f2 $(p \leq 0.001)$. The pictures show part of right hemisections as indicated on the schemes in (D,G) and in (P,S), respectively. Mean values \pm SEM, $n=3$, five sections per level for each embryo $\left(n>635\right.$ cells per condition). ${ }^{* * *}=p \leq 0.001$. Scale bar $=100 \mu \mathrm{m}$.

immunoglobulin-secreting B cells (Corcoran et al., 1993), it is also expressed in the central nervous system during development and in the adult brain (Hatzopoulos et al., 1990; Lillycrop and Latchman, 1992; Stoykova et al., 1992; Camós et al., 2014). A high-resolution in situ hybridization analysis detected Pou $2 f 2$ expression at e13.5 in the midbrain (Thompson et al., 2014), a time point that corresponds to late neurogenesis (Bayer et al., 1995). Consistently, we observed the presence of Pou2f2 in differentiating dI2-dI6 INs of the developing spinal cord. In mouse ES cells undergoing neuronal differentiation, Pou2f 2 could act as a bifunctional regulator of differentiation depending on the predominant isoform (Theodorou et al., 2009), although the isoforms considered are different from those identified in the developing spinal cord (Harris et al., 2019). Pou $2 f 2$ inactivation is associated with neonatal lethality (Corcoran et al., 1993), suggesting its involvement in the development of vital nervous functions. However, its role in neuronal CNS development had not been explored yet. Our analysis of several dIN populations suggested that Pou2f 2 contributes to dIN migration. Potential Pou2f 2 targets in the CNS are currently unknown. Recent studies have uncovered the presence of Pou $2 \mathrm{f} 2$ in several tumors including pancreatic 
and gastric cancers, the latter having a high frequency of invasiveness and metastasis. Interestingly, Pou $2 \mathrm{f} 2$ has been identified in a Nf-kB/Pou2f2/Robo1/Slit2 signaling cascade that promotes metastasis in gastric cancer cells (Wang et al., 2016). In this cascade, Pou2f2 directly regulates the Robo1 gene, a member of the ROBO family, and activates its expression. Several studies point out the importance of the Slit/Robo repulsive signals in neuronal migration (Wu et al., 1999; Causeret et al., 2002; Andrews et al., 2006; Di Meglio et al., 2008). In the developing forebrain, through semaphorin-neuropilin/plexin signaling modulation, Robol guides interneuron migration through the subpallium and into the cortex (HernándezMiranda et al., 2011). Oppositely to Robo1 and Robo2 receptors, Robo3 does not bind Slits but instead interacts with Dcc and Netrin-1. This mechanism of action mediates attraction (Zelina et al., 2014) and, probably through Dcc and Robo3, Netrin-1 promotes dINs migration (Junge et al., 2016). Additionally, Slit/Robo repellent signaling, in parallel with Netrin-1/DCC attractive cues, ensures correct positioning of spinal motor neurons in the ventral horn (Kim et al., 2015). Hence, Pou2f2 may regulate Robo1 expression in dIN populations and thereby promote the production of repulsive guidance cues. Interestingly, in Pou $2 f 2$ mutants, several dIN populations progress deeper than expected in the ventral spinal cord. Secreted Slits are produced by the ventral spinal cord, which makes it a repulsive territory for neurons expressing Robol and Robo2 receptors (Long et al., 2004). In Pou2f2-depleted dINs, Robo1 expression could be downregulated, resulting in altered responsiveness to repellent Slit signals that would authorize a more ventral localization.

Perturbations in neuronal position and/or migration during development result in heavy conditions such as "type I" lissencephaly related to the mislocalization of cortical neurons (Hirotsune et al., 1998; Vallee and Tsai, 2006) or constitute a risk factor for schizophrenia (Tomita et al., 2011). Interestingly, two other POU domain transcription factors, Pou $3 \mathrm{f} 2$ and Pou $3 \mathrm{f} 3$ are suspected to be involved in schizophrenia due to their effect on cortical neuron migration (Potkin et al., 2009; Dominguez et al., 2013). Even if diseases associated with neuronal positioning defects have not been described in the spinal cord yet, it has been recently demonstrated that interneuron localization is critical for proper spinal circuit formation. Indeed, the settlement position of inhibitory V1 IN subsets correlates with their input connectivity (Bikoff et al., 2016). Similarly, stereotypical motor neuron localization is of importance for sensory connection establishment. In fact, proprioceptors target specific DV tiers of the spinal cord without regard to the identity of motor neurons present (Sürmeli et al., 2011). Hence, the adequate position of motor neurons is initially of greater importance for sensorymotor connectivity than their identity. Alterations in dIN settling position also cause impairments of sensory-motor signaling, as demonstrated for Satb2 ${ }^{+}$lamina $\mathrm{V}$ inhibitory sensory relay neurons. Loss of Satb2 resulted in altered medio-lateral position of these dINs, which subsequently affected the proprioceptive innervation on those cells (Hilde et al., 2016). Soma localization may also influence the ingrowth of sensory afferents to the dorsal horn. Indeed, selective depletion of Bcl11a in dINs alters, besides its role in neuron morphogenesis, the traveling of sensory fibers to their spinal targets (John et al., 2012). Other transcription factors are known to influence dIN migration, including Lmx1b and its downstream targets $\operatorname{Drg} 11$ and $R n x$, the depletion of which leads to dI5 aberrant localization (Ding et al., 2004). Here we demonstrated that dINs show aberrant soma settling position when Pou $2 f 2$ is either overexpressed or depleted. Interestingly, the dIN populations affected in the absence of Pou2f 2 are involved either in the modulation of motor neuron activity (Andersson et al., 2012; Bui et al., 2013; Goetz et al., 2015; Satoh et al., 2016) or of ventral premotor interneuron activity (Levine et al., 2014; Hilde et al., 2016), or in the presynaptic inhibition of proprioceptive sensory neuron terminals that hinder sensory inputs onto motor neurons (Betley et al., 2009; Fink et al., 2014; Zhang et al., 2017). Whether alterations in Pou2f2 mutant embryos affect the activity of the sensory-motor circuits of the spinal cord remain to be determined.

\section{DATA AVAILABILITY STATEMENT}

The datasets (microarray comparison of control and

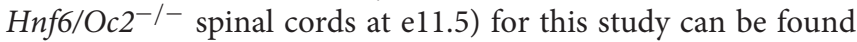
in the GEO repository (accession number: GSE117871; https:// www.ncbi.nlm.nih.gov/geo/query/acc.cgi?acc=GSE117871).

\section{ETHICS STATEMENT}

The animal study was reviewed and approved by Animal Welfare Committee, UCLouvain.

\section{AUTHOR CONTRIBUTIONS}

GM, AH and FC designed the experiments. LC provided critical reagents and contributed to initial discussions. $\mathrm{GM}$ and $\mathrm{AH}$ performed the experiments. BJ contributed to data analyses and all the authors discussed the data. GM and FC drafted the manuscript.

\section{FUNDING}

This work in the FC's laboratory was supported by grants from the "Fonds Spéciaux de Recherche" (FSR) of the Université Catholique de Louvain, by a "Projet de Recherche (PDR)" \#T.0117.13 and an "Equipement (EQP)" funding \#U.N027.14 of the Fonds de la Recherche Scientifique (F.R.S.-FNRS, Belgium), by the "Actions de Recherche Concertées (ARC)" \#10/15-026 of the "Direction Générale de l'Enseignement non Obligatoire et de la Recherche Scientifique-Direction de la Recherche scientifique-Communauté Française de Belgique" and grant by the "Académie Universitaire "Louvain"” and by the Association Belge contre les Maladies neuro-Musculaires (ABMM). LC acknowledges funding from the Australian Government (NHMRC IRIIS and research grants \#637306 and \#575500) and Victorian State Government Operational Infrastructure Support. $\mathrm{GM}$ and $\mathrm{AH}$ hold $\mathrm{PhD}$ grants from the Fonds pour la Recherche dans l'Industrie et l'Agriculture (F.R.S.-FNRS, Belgium), FC is a Senior Research Associate of the F.R.S.-FNRS. 


\section{ACKNOWLEDGMENTS}

We thank members of the NEDI lab for material, technical support and discussions. We are grateful to Dr. K. Kullander for kindly providing the guinea pig

\section{REFERENCES}

Andersson, L. S., Larhammar, M., Memic, F., Wootz, H., Schwochow, D., Rubin, C. J., et al. (2012). Mutations in DMRT3 affect locomotion in horses and spinal circuit function in mice. Nature 488, 642-646. doi: 10.1038/nature11399 Andrews, W., Liapi, A., Plachez, C., Camurri, L., Zhang, J., Mori, S., et al. (2006). Robol regulates the development of major axon tracts and interneuron migration in the forebrain. Development 133, 2243-2252. doi: 10.1242/dev. 02379

Audouard, E., Schakman, O., Ginion, A., Bertrand, L., Gailly, P., and Clotman, F. (2013). The Onecut transcription factor HNF-6 contributes to proper reorganization of Purkinje cells during postnatal cerebellum development. Mol. Cell. Neurosci. 56, 159-168. doi: 10.1016/j.mcn.2013.05.001

Bayer, S. A., Wills, K. V., Triarhou, L. C., and Ghetti, B. (1995). Time of neuron origin and gradients of neurogenesis in midbrain dopaminergic neurons in the mouse. Exp. Brain Res. 105, 191-199. doi: 10.1007/bf00240955

Beguin, S., Crépel, V., Aniksztejn, L., Becq, H., Pelosi, B., PallesiPocachard, E., et al. (2013). An epilepsy-related ARX polyalanine expansion modifies glutamatergic neurons excitability and morphology without affecting gabaergic neurons development. Cereb. Cortex 23, 1484-1494. doi: 10.1093/cercor/bhs138

Bermingham, N. A., Hassan, B. A., Wang, V. Y., Fernandez, M., Banfi, S., Bellen, H. J., et al. (2001). Proprioceptor pathway development is dependent on MATH1. Neuron 30, 411-422. doi: 10.1016/s0896-6273(01) 00305-1

Betley, J. N., Wright, C. V. E., Kawaguchi, Y., Erdélyi, F., Szabó, G., Jessell, T. M., et al. (2009). Stringent specificity in the construction of a GABAergic presynaptic inhibitory circuit. Cell 139, 161-174. doi: 10.1016/j.cell.2009.08.027

Bikoff, J. B., Gabitto, M. I., Rivard, A. F., Drobac, E., MacHado, T. A., Miri, A., et al. (2016). Spinal inhibitory interneuron diversity delineates variant motor microcircuits. Cell 165, 207-219. doi: 10.1016/j.cell.2016.01.027

Borowska, J., Jones, C. T., Deska-Gauthier, D., and Zhang, Y. (2015). V3 interneuron subpopulations in the mouse spinal cord undergo distinctive postnatal maturation processes. Neuroscience 295, 221-228. doi: 10.1016/j. neuroscience.2015.03.024

Borowska, J., Jones, C. T., Zhang, H., Blacklaws, J., Goulding, M., and Zhang, Y. (2013). Functional subpopulations of V3 interneurons in the mature mouse spinal cord. J. Neurosci. 33, 18553-18565. doi: 10.1523/JNEUROSCI.200513.2013

Bui, T. V., Akay, T., Loubani, O., Hnasko, T. S., Jessell, T. M., and Brownstone, R. M. (2013). Circuits for grasping: spinal dI3 interneurons mediate cutaneous control of motor behavior. Neuron 78, 191-204. doi: 10.1016/j.neuron.2013.02.007

Camós, S., Gubern, C., Sobrado, M., Rodríguez, R., Romera, V. G., Moro, M. Á., et al. (2014). Oct-2 transcription factor binding activity and expression up-regulation in rat cerebral ischaemia is associated with a diminution of neuronal damage in vitro. Neuromolecul. Med. 16, 332-349. doi: 10.1007/s12017-013-8279-1

Caspary, T., and Anderson, K. V. (2003). Patterning cell types in the dorsal spinal cord: what the mouse mutants say. Nat. Rev. Neurosci. 4, 289-297. doi: $10.1038 / \mathrm{nrn} 1073$

Catela, C., Shin, M. M., and Dasen, J. S. (2015). Assembly and function of spinal circuits for motor control. Annu. Rev. Cell Dev. Biol. 31, 669-698. doi: 10.1146/annurev-cellbio-100814-125155

Causeret, F., Danne, F., Ezan, F., Sotelo, C., and Bloch-Gallego, E. (2002). Slit antagonizes netrin-1 attractive effects during the migration of inferior olivary neurons. Dev. Biol. 246, 429-440. doi: 10.1006/dbio.2002.0681

Chen, Z. (2019). Common cues wire the spinal cord: axon guidance molecules in spinal neuron migration. Semin. Cell Dev. Biol. 85, 71-77. doi: 10.1016/j. semcdb.2017.12.012 anti Dmrt3 antibody, to Dr. T. Müller for the rabbit or guinea pig anti Foxd3 or anti Lbx1 or anti Lmx1b antibodies, to Dr. J.-F. Brunet for the rabbit anti Phox2a antibody, and to Dr. G. Courtoy for help with image quantifications.

Chopek, J. W., Nascimento, F., Beato, M., Brownstone, R. M., and Zhang, Y. (2018). Sub-populations of spinal V3 interneurons form focal modules of layered pre-motor microcircuits. Cell Rep. 25, 146.e3-156.e3. doi: 10.1016/j. celrep.2018.08.095

Clerc, R. G., Corcoran, L. M., LeBowitz, J. H., Baltimore, D., and Sharp, P. A. (1988). The B-cell-specific Oct-2 protein contains POU box- and homeo box-type domains. Genes Dev. 2, 1570-1581. doi: 10.1101/gad.2.12a.1570

Clotman, F., Jacquemin, P., Plumb-Rudewiez, N., Pierreux, C. E., Van der Smissen, P., Dietz, H. C., et al. (2005). Control of liver cell fate decision by a gradient of TGF $\beta$ signaling modulated by Onecut transcription factors. Genes Dev. 19, 1849-1854. doi: 10.1101/gad.340305

Corcoran, L. M., Karvelas, M., Nossal, G. J. V., Ye, Z. S., Jacks, T., and Baltimore, D. (1993). Oct-2, although not required for early B-cell development, is critical for later B-cell maturation and for postnatal survival. Genes Dev. 7, 570-582. doi: $10.1101 /$ gad.7.4.570

Corcoran, L. M., Koentgen, F., Dietrich, W., Veale, M., and Humbert, P. O. (2004). All known in vivo functions of the Oct-2 transcription factor require the C-terminal protein domain. J. Immunol. 172, 2962-2969. doi: 10.4049/jimmunol.172.5.2962

Del Barrio, M. G., Bourane, S., Grossmann, K., Schüle, R., Britsch, S., O’Leary, D. D. M., et al. (2013). A transcription factor code defines nine sensory interneuron subtypes in the mechanosensory area of the spinal cord. PLoS One 8:e77928. doi: 10.1371/journal.pone.0077928

Di Meglio, T., Nguyen-Ba-Charvet, K. T., Tessier-Lavigne, M., Sotelo, C., and Chédotal, A. (2008). Molecular mechanisms controlling midline crossing by precerebellar neurons. J. Neurosci. 28, 6285-6294. doi: 10.1523/JNEUROSCI. 0078-08.2008

Ding, Y. Q., Yin, J., Kania, A., Zhao, Z. Q., Johnson, R. L., and Chen, Z. F. (2004). Lmxlb controls the differentiation and migration of the superficial dorsal horn neurons of the spinal cord. Development 131, 3693-3703. doi: 10.1242/dev. 01250

Dominguez, M. H., Ayoub, A. E., and Rakic, P. (2013). POU-III transcription factors (Brn1, Brn2, and Oct6) influence neurogenesis, molecular identity, and migratory destination of upper-layer cells of the cerebral cortex. Cereb. Cortex 23, 2632-2643. doi: 10.1093/cercor/bhs252

Espana, A., and Clotman, F. (2012a). Onecut factors control development of the Locus Coeruleus and of the mesencephalic trigeminal nucleus. Mol. Cell. Neurosci. 50, 93-102. doi: 10.1016/j.mcn.2012.04.002

Espana, A., and Clotman, F. (2012b). Onecut transcription factors are required for the second phase of development of the A13 dopaminergic nucleus in the mouse. J. Comp. Neurol. 520, 1424-1441. doi: 10.1002/cne. 22803

Fink, A. J. P., Croce, K. R., Huang, Z. J., Abbott, L. F., Jessell, T. M., and Azim, E. (2014). Presynaptic inhibition of spinal sensory feedback ensures smooth movement. Nature 508, 43-48. doi: 10.1038/nature 13276

Francius, C., and Clotman, F. (2010). Dynamic expression of the Onecut transcription factors HNF-6, OC-2 and OC-3 during spinal motor neuron development. Neuroscience 165, 116-129. doi: 10.1016/j.neuroscience.2009. 09.076

Francius, C., and Clotman, F. (2014). Generating spinal motor neuron diversity: a long quest for neuronal identity. Cell. Mol. Life Sci. 71, 813-829. doi: 10.1007/s00018-013-1398-x

Francius, C., Hidalgo-Figueroa, M., Debrulle, S., Pelosi, B., Rucchin, V., Ronellenfitch, K., et al. (2016). Vsx1 transiently defines an early intermediate V2 interneuron precursor compartment in the mouse developing spinal cord. Front. Mol. Neurosci. 9:145. doi: 10.3389/fnmol.2016. 00145

Goetz, C., Pivetta, C., and Arber, S. (2015). Distinct limb and trunk premotor circuits establish laterality in the spinal cord. Neuron 85, 131-144. doi: 10.1016/j.neuron.2014.11.024 
Gross, M. K., Dottori, M., and Goulding, M. (2002). Lbx1 specifies somatosensory association interneurons in the dorsal spinal cord. Neuron 34, 535-549. doi: 10.1016/s0896-6273(02)00690-6

Gross, M. K., Moran-Rivard, L., Velasquez, T., Nakatsu, M. N., Jagla, K., and Goulding, M. (2000). Lbx1 is required for muscle precursor migration along a lateral pathway into the limb. Development 127, 413-424.

Harris, A., Masgutova, G., Collin, A., Toch, M., Hidalgo-Figueroa, M., Jacob, B., et al. (2019). Onecut factors and Pou2f2 regulate the distribution of V2 interneurons in the mouse developing spinal cord. Front. Cell. Neurosci. 13:184. doi: 10.3389/fncel.2019.00184

Hatzopoulos, A. K., Stoykova, A. S., Erselius, J. R., Goulding, M., Neuman, T., and Gruss, P. (1990). Structure and expression of the mouse Oct2a and Oct2b, two differentially spliced products of the same gene. Development 109 , 349-362.

Hayashi, M., Hinckley, C. A., Driscoll, S. P., Moore, N. J., Levine, A. J., Hilde, K. L., et al. (2018). Graded arrays of spinal and supraspinal V2a interneuron subtypes underlie forelimb and hindlimb motor control. Neuron 97, 869.e5-884.e5. doi: 10.1016/j.neuron.2018.01.023

Helms, A. W., and Johnson, J. E. (2003). Specification of dorsal spinal cord interneurons. Curr. Opin. Neurobiol. 13, 42-49. doi: 10.1016/s09594388(03)00010-2

Hernández-Miranda, L. R., Cariboni, A., Faux, C., Ruhrberg, C., Cho, J. H., Cloutier, J.-F., et al. (2011). Robol regulates semaphorin signaling to guide the migration of cortical interneurons through the ventral forebrain. J. Neurosci. 31, 6174-6187. doi: 10.1523/JNEUROSCI.5464-10.2011

Hernandez-Miranda, L. R., Müller, T., and Birchmeier, C. (2017). The dorsal spinal cord and hindbrain: from developmental mechanisms to functional circuits. Dev. Biol. 432, 34-42. doi: 10.1016/j.ydbio.2016.10.008

Hilde, K. L., Levine, A. J., Hinckley, C. A., Hayashi, M., Montgomery, J. M., Gullo, M., et al. (2016). Satb2 is required for the development of a spinal exteroceptive microcircuit that modulates limb position. Neuron 91, 763-776. doi: 10.1016/j.neuron.2016.07.014

Hinckley, C. A., Alaynick, W. A., Gallarda, B. W., Hayashi, M., Hilde, K. L., Driscoll, S. P., et al. (2015). Spinal locomotor circuits develop using hierarchical rules based on motorneuron position and identity. Neuron 87, 1008-1021. doi: 10.1016/j.neuron.2015.08.005

Hirotsune, S., Fleck, M. W., Gambello, M. J., Bix, G. J., Chen, A., Clark, G. D., et al. (1998). Graded reduction of Pafah1b1 (Lis1) activity results in neuronal migration defects and early embryonic lethality. Nat. Genet. 19, 333-339. doi: $10.1038 / 1221$

Hodson, D. J., Shaffer, A. L., Xiao, W., Wright, G. W., Schmitz, R., Phelan, J. D., et al. (2016). Regulation of normal B-cell differentiation and malignant B-cell survival by OCT2. Proc. Natl. Acad. Sci. U S A 113, E2039-E2046. doi: 10.1073/pnas.1600557113

Jacquemin, P., Durviaux, S. M., Jensen, J., Godfraind, C., Gradwohl, G., Guillemot, F., et al. (2000). Transcription factor hepatocyte nuclear factor 6 regulates pancreatic endocrine cell differentiation and controls expression of the proendocrine gene ngn3. Mol. Cell. Biol. 20, 4445-4454. doi: 10.1128/mcb. 20.12.4445-4454.2000

Jacquemin, P., Lannoy, V. J., Rousseau, G. G., and Lemaigre, F. P. (1999). OC-2, a novel mammalian member of the ONECUT class of homeodomain transcription factors whose function in liver partially overlaps with that of hepatocyte nuclear factor-6. J. Biol. Chem. 274, 2665-2671. doi: 10.1074/jbc. 274.5.2665

Jacquemin, P., Lemaigre, F. P., and Rousseau, G. G. (2003). The Onecut transcription factor HNF-6 (OC-1) is required for timely specification of the pancreas and acts upstream of Pdx-1 in the specification cascade. Dev. Biol. 258, 105-116. doi: 10.1016/s0012-1606(03)00115-5

John, A., Brylka, H., Wiegreffe, C., Simon, R., Liu, P., Jüttner, R., et al. (2012). Bcll1a is required for neuronal morphogenesis and sensory circuit formation in dorsal spinal cord development. Development 139, 1831-1841. doi: 10.1242/dev.072850

Junge, H. J., Yung, A. R., Goodrich, L. V., and Chen, Z. (2016). Netrin1/DCC signaling promotes neuronal migration in the dorsal spinal cord. Neural Dev. 11:19. doi: 10.1186/s13064-016-0074-x

Kabayiza, K. U., Masgutova, G., Harris, A., Rucchin, V., Jacob, B., and Clotman, F. (2017). The onecut transcription factors regulate differentiation and distribution of dorsal interneurons during spinal cord development. Front. Mol. Neurosci. 10:157. doi: 10.3389/fnmol.2017. 00157

Kim, M., Fontelonga, T., Roesener, A. P., Lee, H., Gurung, S., Mendonca, P. R. F., et al. (2015). Motor neuron cell bodies are actively positioned by Slit/Robo repulsion and Netrin/DCC attraction. Dev. Biol. 399, 68-79. doi: 10.1016/j. ydbio.2014.12.014

König, H., Pfisterer, P., Corcoran, L. M., and Wirth, T. (1995). Identification of CD36 as the first gene dependent on the b-cell differentiation factor Oct-2. Genes Dev. 9, 1598-1607. doi: 10.1101/gad.9.13.1598

Ladle, D. R., Pecho-Vrieseling, E., and Arber, S. (2007). Assembly of motor circuits in the spinal cord: driven to function by genetic and experience-dependent mechanisms. Neuron 56, 270-283. doi: 10.1016/j.neuron.2007.09.026

Lai, H. C., Seal, R. P., and Johnson, J. E. (2016). Making sense out of spinal cord somatosensory development. Development 143, 3434-3448. doi: 10.1242/dev. 139592

Landry, C., Clotman, F., Hioki, T., Oda, H., Picard, J. J., Lemaigre, F. P., et al. (1997). HNF-6 is expressed in endoderm derivatives and nervous system of the mouse embryo and participates to the cross-regulatory network of liverenriched transcription factors. Dev. Biol. 192, 247-257. doi: 10.1006/dbio. 1997.8757

Lanuza, G. M., Gosgnach, S., Pierani, A., Jessell, T. M., and Goulding, M. (2004). Genetic identification of spinal interneurons that coordinate left-right locomotor activity necessary for walking movements. Neuron 42, 375-386. doi: 10.1016/s0896-6273(04)00249-1

Leggere, J. C., Saito, Y., Darnell, R. B., Tessier-Lavigne, M., Junge, H. J., and Chen, Z. (2016). NOVA regulates Dcc alternative splicing during neuronal migration and axon guidance in the spinal cord. Elife 5:e14264. doi: 10.7554/eLife.14264

Lemaigre, F. P., Durviaux, S. M., Truong, O., Lannoy, V. J., Hsuan, J. J., and Rousseau, G. G. (1996). Hepatocyte nuclear factor 6, a transcription factor that contains a novel type of homeodomain and a single cut domain. Proc. Natl. Acad. Sci. U S A 93, 9460-9464. doi: 10.1073/pnas.93.18.9460

Levine, A. J., Hinckley, C. A., Hilde, K. L., Driscoll, S. P., Poon, T. H., Montgomery, J. M., et al. (2014). Identification of a cellular node for motor control pathways. Nat. Neurosci. 17, 586-593. doi: 10.1038/nn.3675

Lillycrop, K. A., and Latchman, D. S. (1992). Alternative splicing of the Oct-2 transcription factor RNA is differentially regulated in neuronal cells and B cells and results in protein isoforms with opposite effects on the activity of octamer/TAATGARAT- containing promoters. J. Biol. Chem. 267, 24960-24965.

Long, H., Sabatier, C., Ma, L., Plump, A., Yuan, W., Ornitz, D. M., et al. (2004). Conserved roles for slit and robo proteins in midline commissural axon guidance. Neuron 42, 213-223. doi: 10.1016/s0896-6273(04)00179-5

Lu, D. C., Niu, T., and Alaynick, W. A. (2015). Molecular and cellular development of spinal cord locomotor circuitry. Front. Mol. Neurosci. 8:25. doi: 10.3389/fnmol.2015.00025

Marín, O., Valiente, M., Ge, X., and Tsai, L. H. (2010). Guiding neuronal cell migrations. Cold Spring Harb. Perspect. Biol. 2:a001834. doi: 10.1101/cshperspect.a001834.

Müller, T., Brohmann, H., Pierani, A., Heppenstall, P. A., Lewin, G. R., Jessell, T. M., et al. (2002). The homeodomain factor lbxl distinguishes two major programs of neuronal differentiation in the dorsal spinal cord. Neuron 34, 551-562. doi: 10.1016/s0896-6273(02)00689-x

Palmesino, E., Rousso, D. L., Kao, T. J., Klar, A., Laufer, E., Uemura, O., et al. (2010). Foxp1 and Lhxl coordinate motor neuron migration with axon trajectory choice by gating reelin signalling. PLoS Biol. 8:e1000446. doi: 10.1371/journal.pbio.1000446

Pelosi, B., Migliarini, S., Pacini, G., Pratelli, M., and Pasqualetti, M. (2014). Generation of Pet1210-Cre transgenic mouse line reveals non-serotonergic expression domains of Pet1 both in CNS and periphery. PLoS One 9:e104318. doi: 10.1371/journal.pone.0104318

Pierreux, C. E., Vanhorenbeeck, V., Jacquemin, P., Lemaigre, F. P., and Rousseau, G. G. (2004). The transcription factor hepatocyte nuclear factor6/Onecut-1 controls the expression of its paralog Onecut-3 in developing mouse endoderm. J. Biol. Chem. 279, 51298-51304. doi: 10.1074/jbc. m409038200

Pillai, A., Mansouri, A., Behringer, R., Westphal, H., and Goulding, M. (2007). Lhx1 and Lhx5 maintain the inhibitory-neurotransmitter status 
of interneurons in the dorsal spinal cord. Development 134, 357-366. doi: 10.1242/dev.02717

Potkin, S. G., Turner, J. A., Guffanti, G., Lakatos, A., Fallon, J. H., Nguyen, D. D., et al. (2009). A genome-wide association study of schizophrenia using brain activation as a quantitative phenotype. Schizophr. Bull. 35, 96-108. doi: 10.1093/schbul/sbn155

Rosenberg, A. B., Roco, C. M., Muscat, R. A., Kuchina, A., Sample, P., Yao, Z., et al. (2018). Single-cell profiling of the developing mouse brain and spinal cord with split-pool barcoding. Science 360, 176-182. doi: 10.1126/science.aam8999

Roy, A., Francius, C., Rousso, D. L., Seuntjens, E., Debruyn, J., Luxenhofer, G., et al. (2012). Onecut transcription factors act upstream of isll to regulate spinal motoneuron diversification. Development 139, 3109-3119. doi: 10.1242/dev. 078501

Satoh, D., Pudenz, C., and Arber, S. (2016). Context-dependent gait choice elicited by EphA4 mutation in Lbx1 spinal interneurons. Neuron 89, 1046-1058. doi: 10.1016/j.neuron.2016.01.033

Schnerwitzki, D., Perry, S., Ivanova, A., Caixeta, F. V., Cramer, P., Günther, S., et al. (2018). Neuron-specific inactivation of Wt1 alters locomotion in mice and changes interneuron composition in the spinal cord. Life Sci. Alliance 1:e201800106. doi: 10.26508/lsa.201800106

Seufert, D. W., Prescott, N. L., and El-Hodiri, H. M. (2005). Xenopus aristalessrelated homeobox (xArx) gene product functions as both a transcriptional activator and repressor in forebrain development. Dev. Dyn. 232, 313-324. doi: $10.1002 /$ dvdy.20234

Stam, F. J., Hendricks, T. J., Zhang, J., Geiman, E. J., Francius, C., Labosky, P. A., et al. (2012). Renshaw cell interneuron specialization is controlled by a temporally restricted transcription factor program. Development 139, 179-190. doi: $10.1242 /$ dev.071134

Stoykova, A. S., Sterrer, S., Erselius, J. R., Hatzopoulos, A. K., and Gruss, P. (1992). Mini-Oct and Oct-2c: two novel, functionally diverse murine Oct-2 gene products are differentially expressed in the CNS. Neuron 8, 541-558. doi: 10.1016/0896-6273(92)90282-i

Sürmeli, G., Akay, T., Ippolito, G. C., Tucker, P. W., and Jessell, T. M. (2011). Patterns of spinal sensory-motor connectivity prescribed by a dorsoventral positional template. Cell 147, 653-665. doi: 10.1016/j.cell.2011.10.012

Sweeney, L. B., Bikoff, J. B., Gabitto, M. I., Brenner-Morton, S., Baek, M., Yang, J. H., et al. (2018). Origin and segmental diversity of spinal inhibitory interneurons. Neuron 97, 341-355. doi: 10.1016/j.neuron.2017.12.029

Theodorou, E., Dalembert, G., Heffelfinger, C., White, E., Weissman, S., Corcoran, L., et al. (2009). A high throughput embryonic stem cell screen identifies Oct-2 as a bifunctional regulator of neuronal differentiation. Genes Dev. 23, 575-588. doi: 10.1101/gad.1772509

Thompson, C. L., Ng, L., Menon, V., Martinez, S., Lee, C. K., Glattfelder, K., et al. (2014). A high-resolution spatiotemporal atlas of gene expression of the developing mouse brain. Neuron 83, 309-323. doi: 10.1016/j.neuron.2014. 05.033

Tomita, K., Kubo, K., Ishii, K., and Nakajima, K. (2011). Disrupted-inschizophrenia-1 (Disc1) is necessary for migration of the pyramidal neurons during mouse hippocampal development. Hum. Mol. Genet. 20, 2834-2845. doi: 10.1093/hmg/ddr194

Tripodi, M., Stepien, A. E., and Arber, S. (2011). Motor antagonism exposed by spatial segregation and timing of neurogenesis. Nature 479, 61-66. doi: $10.1038 /$ nature 10538

Tyurina, O. V., Guner, B., Popova, E., Feng, J., Schier, A. F., Kohtz, J. D., et al. (2005). Zebrafish Gli3 functions as both an activator and a repressor in Hedgehog signaling. Dev. Biol. 277, 537-556. doi: 10.1016/j.ydbio.2004.10.003

Vallee, R. B., and Tsai, J. W. (2006). The cellular roles of the lissencephaly gene LIS1 and what they tell us about brain development. Genes Dev. 20, 1384-1393. doi: $10.1101 /$ gad.1417206

Vanhorenbeeck, V., Jacquemin, P., Lemaigre, F. P., and Rousseau, G. G. (2002). OC-3, a novel mammalian member of the ONECUT class of transcription factors. Biochem. Biophys. Res. Commun. 292, 848-854. doi: 10.1006/bbrc. 2002.6760

Wang, S. M., Tie, J., Wang, W. L., Hu, S. J., Yin, J. P., Yi, X. F., et al. (2016) POU2F2-oriented network promotes human gastric cancer metastasis. Gut 65, 1427-1438. doi: 10.1136/gutjnl-2014-308932

Wu, W., Wong, K., Chen, J. H., Jiang, Z. H., Dupuls, S., Wu, J. Y., et al. (1999). Directional guidance of neuronal migration in the olfactory system by the protein Slit. Nature 400, 331-336. doi: 10.1038/22477

Zelina, P., Blockus, H., Zagar, Y., Péres, A., Friocourt, F., Wu, Z., et al. (2014). Signaling switch of the axon guidance receptor Robo3 during vertebrate evolution. Neuron 84, 1258-1272. doi: 10.1016/j.neuron.2014.11.004

Zhang, J., Weinrich, J. A. P., Russ, J. B., Comer, J. D., Bommareddy, P. K., DiCasoli, R. J., et al. (2017). A role for dystonia-associated genes in spinal GABAergic interneuron circuitry. Cell Rep. 21, 666-678. doi: 10.1016/j.celrep. 2017.09.079

Conflict of Interest: The authors declare that the research was conducted in the absence of any commercial or financial relationships that could be construed as a potential conflict of interest.

Copyright (c) 2019 Masgutova, Harris, Jacob, Corcoran and Clotman. This is an open-access article distributed under the terms of the Creative Commons Attribution License (CC BY). The use, distribution or reproduction in other forums is permitted, provided the original author(s) and the copyright owner(s) are credited and that the original publication in this journal is cited, in accordance with accepted academic practice. No use, distribution or reproduction is permitted which does not comply with these terms. 